\title{
Some trapezoid and midpoint type inequalities via fractional $(p, q)$-calculus
}

\author{
Pheak Neang ${ }^{1}$ (D), Kamsing Nonlaopon ${ }^{1 *} \mathbb{D}$, Jessada Tariboon ${ }^{2}$, Sotiris K. Ntouyas ${ }^{3,4}$ (D) and \\ Praveen Agarwal ${ }^{5,6}$ (D)
}

"Correspondence: nkamsi@kku.ac.th ${ }^{1}$ Department of Mathematics, Khon Kaen University, 40002 Khon Kaen, Thailand

Full list of author information is available at the end of the article

\section{Springer}

\begin{abstract}
Fractional calculus is the field of mathematical analysis that investigates and applies integrals and derivatives of arbitrary order. Fractional $q$-calculus has been investigated and applied in a variety of research subjects including the fractional $q$-trapezoid and $q$-midpoint type inequalities. Fractional $(p, q)$-calculus on finite intervals, particularly the fractional $(p, q)$-integral inequalities, has been studied. In this paper, we study two identities for continuous functions in the form of fractional $(p, q)$-integral on finite intervals. Then, the obtained results are used to derive some fractional $(p, q)$-trapezoid and $(p, q)$-midpoint type inequalities.
\end{abstract}

MSC: 05A30; 26D10; 26D15; 26A33

Keywords: Trapezoid type inequalities; Midpoint type inequalities; Quantum calculus; $q$-shifting operator; $(p, q)$-calculus; Fractional $(p, q)$-integral; Fractional $(p, q)$-integral inequalities

\section{Introduction}

The ordinary calculus of Newton and Leibniz is well known to be investigated extensively and intensively to produce a large number of related formulas and properties as well as applications in a variety of fields ranging from natural sciences to social sciences. In the early eighteenth century, the well-known mathematician Leonhard Euler (1707-1783) established quantum calculus or $q$-calculus, which is the study of calculus without limits, in the way of Newton's work for infinite series. Later, F. H. Jackson initiated a study of $q$ calculus in a symmetrical manner in 1910 and introduced $q$-derivative and $q$-integral in [1], see [2] for more details.

Many physical and mathematical problems have led to the necessity of studying $q$ calculus; for instance, Fock [3] studied the symmetry of hydrogen atoms using the $q$ difference equation. In addition, in modern mathematical analysis, $q$-calculus has lots of applications such as combinatorics, orthogonal polynomials, basic hypergeometric functions, number theory, quantum theory, mechanics, and theory of relativity, see also [4-24] and the references cited therein. The book by Kac and Cheung [25] covers the basic theoretical concepts of $q$-calculus.

(c) The Author(s) 2021. This article is licensed under a Creative Commons Attribution 4.0 International License, which permits use sharing, adaptation, distribution and reproduction in any medium or format, as long as you give appropriate credit to the original author(s) and the source, provide a link to the Creative Commons licence, and indicate if changes were made. The images or other third party material in this article are included in the article's Creative Commons licence, unless indicated otherwise in a credit line to the material. If material is not included in the article's Creative Commons licence and your intended use is not permitted by statutory regulation or exceeds the permitted use, you will need to obtain permission directly from the copyright holder. To view a copy of this licence, visit http://creativecommons.org/licenses/by/4.0/. 
As one of the major driving forces behind the modern approach of real analysis, inequalities have played a vital role in almost all branches of mathematics along with other fields of science. In 2015, Noor et al. [26] established $q$-analogue of classical integral identity to obtain $q$-trapezoid type inequalities for convex functions. Moreover, in 2016, Necmettin, Mehmet, and İmdat [27] proved the correctness of left part of $q$-Hermite-Hadamard and gave some $q$-midpoint type integral inequalities through $q$-differentiable convex function and $q$-differentiable quasi-convex functions. With these results, many researchers have extended some important topics of $q$-calculus together with applications in many fields, such as $q$-integral inequalities, see [28-37] for more details.

Since the exploration has been continued to generalize the existing results through creative thoughts and novel techniques of fractional calculus, in 2015, Tariboon, Ntouyas, and Agarwal [38] proposed a new $q$-shifting operator ${ }_{a} \Phi_{q}(m)=q m+(1-q) a$ for studying new concepts of fractional $q$-calculus. In 2016, Sudsutad, Ntouyas, and Tariboon [39] studied some fractional $q$-integral inequalities. In 2020, Kunt and Aljasem [40] proved RiemannLiouville fractional $q$-trapezoid and $q$-midpoint type inequalities for convex functions. Furthermore, in 2021, Neang et al. [41] introduced fractional $(p, q)$-calculus on finite intervals and proved some well-known integral inequalities.

In 2018, as one of the most attractive areas, Kunt et al. [42] proved ( $p, q)$-HermiteHadamard inequalities and gave some $(p, q)$-midpoint type integral inequalities via $(p, q)$ differentiable convex and $(p, q)$-differentiable quasi-convex functions. In 2019, Latif et al. [43] proved some $(p, q)$-trapezoid integral inequalities for convex and quasi-convex functions. Based on these results, many authors have generalized and developed $(p, q)$ calculus, which is used efficiently in many fields, and some results on the study of $(p, q)$ calculus can be found in [44-71].

Motivated by some of the above studies and applications, in this paper, we study two identities for continuous functions in the form of fractional $(p, q)$-integral on finite intervals. Then, the obtained results are used to derive some fractional $(p, q)$-trapezoid and $(p, q)$-midpoint type inequalities.

\section{Preliminaries}

In this section, we recall some well-known facts on fractional $(p, q)$-calculus, which can be found in $[10,11,38,53,55]$. Throughout this paper, let $[a, b] \subset \mathbb{R}$ be an interval with $a<b$, and $0<q<p \leq 1$ be constants,

$$
\begin{aligned}
& {[k]_{p, q}=\left\{\frac{p^{k}-q^{k}}{p-q}, \quad k \in \mathbb{N},\right.} \\
& {[k]_{p, q} != \begin{cases}{[k]_{p, q}[k-1]_{p, q} \cdots[1]_{p, q}=\prod_{i=1}^{k} \frac{p^{i}-q^{i}}{p-q},} & k \in \mathbb{N}, \\
1, & k=0 .\end{cases} }
\end{aligned}
$$

Property 2.1 ([38]) Let ${ }_{a} \Phi_{q}(m)=q m+(1-q) a$. For any $m, n \in \mathbb{R}$ and for all positive integers $j, k$, we have

(i) ${ }_{a} \Phi_{q}^{k}(m)={ }_{a} \Phi_{q^{k}}(m)$;

(ii) ${ }_{a} \Phi_{q}^{j}\left({ }_{a} \Phi_{q}^{k}(m)\right)={ }_{a} \Phi_{q}^{k}\left({ }_{a} \Phi_{q}^{j}(m)\right)={ }_{a} \Phi_{q}^{j+k}(m)$;

(iii) ${ }_{a} \Phi_{q}(a)=a$;

(iv) ${ }_{a} \Phi_{q}^{k}(m)-a=q^{k}(m-a)$;

(v) $m-{ }_{a} \Phi_{q}^{k}(m)=\left(1-q^{k}\right)(m-a)$; 
(vi) ${ }_{a} \Phi_{q}^{k}(m)=m_{a / m} \Phi_{q}^{k}(1)$ for $m \neq 0$;

(vii) ${ }_{a} \Phi_{q}(m)-{ }_{a} \Phi_{q}^{k}(n)=q\left(m-{ }_{a} \Phi_{q}^{k-1}(n)\right)$.

Property 2.2 ([38]) For any $\gamma, n, m \in \mathbb{R}$ with $n \neq a$ and $k \in \mathbb{N} \cup\{0\}$, we have

(i) $(n-m)_{a}^{(k)}=(n-a)^{k}\left(\frac{m-a}{n-a} ; q\right)_{k}$;

(ii) $(n-m)^{(\gamma)}{ }_{a}=(n-a)^{\gamma} \prod_{i=0}^{\infty} \frac{1-\frac{m-a}{n-a} q^{i}}{1-\frac{m-a}{n-a} q^{\gamma+i}}=(n-a)^{\gamma} \frac{\left(\frac{m-a}{n-a} ; q\right)_{\infty}}{\left(\frac{m-a}{n-a} q^{\gamma} ; q\right)_{\infty}}$;

(iii) $\left(n-{ }_{a} \Phi_{q}^{k}(n)\right)_{a}^{\gamma}=(n-a)^{\gamma} \frac{\left(q^{k} ; q\right)_{\infty}}{\left(q^{\gamma+k} ; q\right)_{\infty}}$.

For $m, n \in \mathbb{R}$, the $(p, q)$-analogue of the power function ${ }_{a}(m-n)_{p, q}^{k}$ with $k \in \mathbb{N} \cup\{0\}$ is defined follows:

$$
\begin{aligned}
& a(m-n)_{p, q}^{(0)}:=1, \quad{ }_{a}(m-n)_{p, q}^{(k)}:=\prod_{i=0}^{k-1}\left({ }_{a} \Phi_{p}^{i}(m)-{ }_{a} \Phi_{q}^{i}(n)\right), \\
& { }_{a}(m-n)_{p, q}^{(k)}=(m-a)^{k} \prod_{i=0}^{k-1} p^{i}\left(1-\left(\frac{n-a}{m-a}\right)\left(\frac{q}{p}\right)^{i}\right) .
\end{aligned}
$$

More generally, if $\alpha \in \mathbb{R}$, then

$$
{ }_{a}(m-n)_{p, q}^{(\alpha)}=(m-a)^{\alpha} \prod_{i=0}^{\infty} \frac{p^{i}}{p^{\alpha+i}} \frac{1-\left(\frac{n-a}{m-a}\right)\left(\frac{q}{p}\right)^{i}}{1-\left(\frac{n-a}{m-a}\right)\left(\frac{q}{p}\right)^{\alpha+i}},
$$

or

$$
a_{a}(m-n)_{p, q}^{(\alpha)}=(m-a)^{\alpha} p^{\left(\begin{array}{c}
\alpha \\
2
\end{array}\right)} \prod_{i=0}^{\infty} \frac{1-\left(\frac{n-a}{m-a}\right)\left(\frac{q}{p}\right)^{i}}{1-\left(\frac{n-a}{m-a}\right)\left(\frac{q}{p}\right)^{\alpha+i}} .
$$

Property 2.3 ([41]) For $\alpha>0$, the following formulas hold:

(i) ${ }_{a} \Phi_{q / p}^{k}(m)-a=\left(\frac{q}{p}\right)^{k}(m-a)$;

(ii) $a\left(m-{ }_{a} \Phi_{q / p}^{k}(m)\right)_{p, q}^{(\alpha)}=(m-a)^{\alpha} \prod_{i=0}^{\infty} \frac{p^{i}}{p^{\alpha+i}} \frac{1-\left(\frac{q}{p}\right)^{k}\left(\frac{q}{p}\right)^{i}}{1-\left(\frac{q}{p}\right)^{k}\left(\frac{q}{p}\right)^{(\alpha+i)}}=(m-a)^{\alpha}\left(1-\left(\frac{q}{p}\right)^{k}\right)_{p, q}^{(\alpha)}$.

Definition 2.1 ([72]) If $f:[a, b] \rightarrow \mathbb{R}$ is a continuous function, then the $(p, q)$-derivative of $f$ on $\left[a, \frac{1}{p}(b-a)+a\right]$ at $x$ is defined by

$$
\begin{aligned}
& { }_{a} D_{p, q} f(x)=\frac{f(p x+(1-p) a)-f(q x+(1-q) a)}{(p-q)(x-a)}, \quad x \neq a, \\
& { }_{a} D_{p, q} f(a)=\lim _{x \rightarrow a} D_{p, q} f(x) .
\end{aligned}
$$

Obviously, a function $f$ is $(p, q)$-differentiable on $\left[a, \frac{1}{p}(b-a)+a\right]$ if ${ }_{a} D_{p, q} f(x)$ exists for all $x \in\left[a, \frac{1}{p}(b-a)+a\right]$. In Definition 2.1, if $a=0$, then ${ }_{0} D_{p, q} f=D_{p, q} f$, where $D_{p, q} f$ is defined by

$$
D_{p, q} f(x)=\frac{f(p x)-f(q x)}{(p-q) x}, \quad x \neq 0 .
$$

Furthermore, if $p=1$ in (2.7), then it reduces to $D_{q} f$, which is $q$-derivative of the function $f$, see $[25,73]$ for more details. 
Definition 2.2 ([72]) If $f:[a, b] \rightarrow \mathbb{R}$ is a continuous function, then the $(p, q)$-integral is defined by

$$
\int_{a}^{x} f(t){ }_{a} d_{p, q} t=(p-q)(x-a) \sum_{n=0}^{\infty} \frac{q^{n}}{p^{n+1}} f\left(\frac{q^{n}}{p^{n+1}} x+\left(1-\frac{q^{n}}{p^{n+1}}\right) a\right)
$$

for $x \in\left[a, \frac{1}{p}(b-a)+a\right]$. If $a=0$ and $p=1$ in (2.8), then we have the classical $q$-integral, see [25] for more details.

Theorem 2.1 ([72]) The following formulas hold for $t \in[a, b]$ :

(i) ${ }_{a} D_{p, q} \int_{a}^{t} f(s)_{a} d_{p, q} s=f(t)$;

(ii) $\int_{a}^{b}{ }_{a} D_{p, q} f(s)_{a} d_{p, q} s=f(t)-f(a)$;

(iii) $\int_{c}^{t}{ }_{a} D_{p, q} f(s){ }_{a} d_{p, q} s=f(t)-f(c)$ for $c \in(a, t)$.

Theorem 2.2 ([72]) If $f, g:[a, b] \rightarrow \mathbb{R}$ are continuous functions and $\lambda \in \mathbb{R}$, then the following formulas hold:

(i) $\int_{a}^{t}[f(s)+g(s)]_{a} d_{p, q} s=\int_{a}^{t} f(s)_{a} d_{p, q} s+\int_{a}^{t} g(s)_{a} d_{p, q} s$;

(ii) $\int_{a}^{t} \lambda f(s)_{a} d_{p, q} s=\lambda \int_{a}^{t} f(s)_{a} d_{p, q} s$;

(iii) $\int_{a}^{t} f(p s+(1-p) a)_{a} D_{p, q} g(s)_{a} d_{p, q} s=\left.(f g)(s)\right|_{a} ^{t}-\int_{a}^{t} g(q s+(1-q) a)_{a} D_{p, q}(f(s))_{a} d_{p, q} s$.

For $t \in \mathbb{R} \backslash\{0,-1,-2, \ldots\}$, the $(p, q)$-gamma function is defined by

$$
\Gamma_{p, q}(t)=\frac{(p-q)_{p, q}^{(t-1)}}{(p-q)^{t-1}}
$$

and an equivalent definition of (2.9) is given in [56] as

$$
\Gamma_{p, q}(t)=p^{\frac{t(t-1)}{2}} \int_{0}^{\infty} x^{t-1} E_{p, q}^{-q x} d_{p, q} x
$$

where

$$
E_{p, q}^{-q x}=\sum_{n=0}^{\infty} \frac{q^{\left(\begin{array}{c}
n \\
2
\end{array}\right)}}{[n]_{p, q}}(-q x)^{n}
$$

Obviously, $\Gamma_{p, q}(t+1)=[t]_{p, q} \Gamma_{p, q}(t)$. For $s, t>0$, the definition of the $(p, q)$-beta function is defined by

$$
B_{p, q}(s, t)=\int_{0}^{1} u^{s-1}{ }_{0}\left(1-{ }_{0} \Phi_{q}(u)\right)_{p, q}^{(t-1)}{ }_{0} d_{p, q} u
$$

and (2.11) can also be written as

$$
B_{p, q}(s, t)=p^{(t-1)(2 s+t-2) / 2} \frac{\Gamma_{p, q}(s) \Gamma_{p, q}(t)}{\Gamma_{p, q}(s+t)},
$$

see $[74,75]$ for more details. 
Definition 2.3 ([41]) Let $f$ be a function defined on $[a, b]$, and let $\alpha>0$. The RiemannLiouville fractional $(p, q)$-integral is defined by

$$
\begin{aligned}
& \left({ }_{a} I_{p, q}^{\alpha} f\right)(t) \\
& =\frac{1}{p^{\left(\begin{array}{c}
\alpha \\
2
\end{array}\right)} \Gamma_{p, q}(\alpha)} \int_{a}^{t}{ }_{a}\left(t-{ }_{a} \Phi_{q}(s)\right)_{p, q}^{(\alpha-1)} f\left(\frac{s}{p^{\alpha-1}}+\left(1-\frac{1}{p^{\alpha-1}}\right) a\right){ }_{a} d_{p, q} s \\
& \quad=\frac{(p-q)(t-a)}{p^{\left(\begin{array}{c}
\alpha \\
2
\end{array}\right)} \Gamma_{p, q}(\alpha)} \sum_{n=0}^{\infty} \frac{q^{n}}{p^{n+1}} a\left(t-{ }_{a} \Phi_{q / p}^{n+1}(t)\right)_{p, q}^{(\alpha-1)} f\left(\frac{q^{n}}{p^{\alpha+n}} t+\left(1-\frac{q^{n}}{p^{\alpha+n}}\right) a\right)
\end{aligned}
$$

for $t \in\left[a, p^{\alpha}(b-a)+a\right]$.

Theorem 2.3 ([41]) If $:[a, b] \rightarrow \mathbb{R}$ is a convex differentiable function and $\alpha>0$, then we have

$$
\begin{aligned}
f\left(\frac{\left([\alpha+1]_{p, q}-p^{\alpha}\right) a+p^{\alpha} b}{[\alpha+1]_{p, q}}\right) & \leq \frac{\Gamma_{p, q}(\alpha+1)}{p^{\alpha^{2}}(b-a)^{\alpha}}\left({ }_{a} I_{p, q}^{\alpha} f(s)\right)\left(p^{\alpha} b+\left(1-p^{\alpha}\right) a\right) \\
& \leq \frac{\left([\alpha+1]_{p, q}-p^{\alpha}\right) f(a)+p^{\alpha} f(b)}{[\alpha+1]_{p, q}}
\end{aligned}
$$

\section{Main results}

In this section, we give two identities for continuous functions in the form of fractional Riemann-Liouville $(p, q)$-integral type which will be used to prove the fractional Riemann-Liouville $(p, q)$-trapezoid and $(p, q)$-midpoint type inequalities.

Lemma 3.1 Let $f:[a, b] \rightarrow \mathbb{R}$ be a continuous function and $\alpha>0$. If ${ }_{a} D_{p, q} f$ is $(p, q)$ integrable on $\left(a, \frac{1}{p}(b-a)+a\right)$, then the following equality holds:

$$
\begin{aligned}
& \frac{\Gamma_{p, q}(\alpha+1)}{p^{\alpha^{2}}(b-a)^{\alpha}}\left({ }_{a} I_{p, q}^{\alpha} f\right)\left(p^{\alpha} b+\left(1-p^{\alpha}\right) a\right)-\frac{\left([\alpha+1]_{p, q}-p^{\alpha}\right) f(a)+p^{\alpha} f(b)}{[\alpha+1]_{p, q}} \\
& \quad=\frac{(b-a)}{[\alpha+1]_{p, q}} \int_{0}^{1}\left([\alpha+1]_{p, q}\left(1-\Phi_{q}(t)\right)_{p, q}^{\alpha}-p^{\alpha}\right)_{a} D_{p, q} f((1-t) a+t b)_{0} d_{p, q} t .
\end{aligned}
$$

Proof By simple computation and using Definition 2.3, we have

$$
\begin{aligned}
A_{1}= & \frac{b-a}{p^{\left(\begin{array}{c}
\alpha \\
2
\end{array}\right)}} \int_{0}^{1}\left(1-{ }_{0} \Phi_{q}(t)\right)_{p, q}^{(\alpha)} a D_{p, q} f((1-t) a+t b){ }_{0} d_{p, q} t \\
= & \frac{b-a}{p^{\left(\begin{array}{c}
\alpha \\
2
\end{array}\right)}} \int_{0}^{1}\left(1-{ }_{0} \Phi_{q}(t)\right)_{p, q}^{(\alpha)} \frac{f((1-p t) a+p t b)-f((1-q t) a+q t b)}{(p-q)(b-a) t}{ }_{0} d_{p, q} t \\
= & \frac{1}{p^{\left(\begin{array}{c}
\alpha \\
2
\end{array}\right)}(p-q)} \int_{0}^{1}\left(1-{ }_{0} \Phi_{q}(t)\right)_{p, q}^{(\alpha)} \frac{f((1-p t) a+p t b)}{t}{ }_{0} d_{p, q} t \\
& -\frac{1}{p^{\left(\begin{array}{c}
\alpha \\
2
\end{array}\right)}(p-q)} \int_{0}^{1}\left(1-{ }_{0} \Phi_{q}(t)\right)_{p, q}^{(\alpha)} \frac{f((1-q t) a+q t b)}{t}{ }_{0} d_{p, q} t \\
= & \frac{1}{p^{\left(\begin{array}{c}
\alpha \\
2
\end{array}\right)}} \sum_{n=0}^{\infty} \frac{q^{n}}{p^{n+1}}\left(1-{ }_{0} \Phi_{q / p}^{n+1}(1)\right)_{p, q}^{(\alpha)} \frac{f\left(\left(1-{ }_{0} \Phi_{q / p}^{n}(1)\right) a+{ }_{0} \Phi_{q / p}^{n}(1) b\right)}{\frac{q^{n}}{p^{n+1}}}
\end{aligned}
$$




$$
\begin{aligned}
& -\frac{1}{p^{\left(\begin{array}{c}
\alpha \\
2
\end{array}\right)}} \sum_{n=0}^{\infty} \frac{q^{n}}{p^{n+1}}\left(1-{ }_{0} \Phi_{q / p}^{n+1}(1)\right)_{p, q}^{(\alpha)} \frac{f\left(\left(1-{ }_{0} \Phi_{q / p}^{n+1}(1)\right) a+{ }_{0} \Phi_{q / p}^{n+1}(1) b\right)}{\frac{q^{n}}{p^{n+1}}} \\
& =\sum_{n=0}^{\infty} \frac{\left(\left(\frac{q}{p}\right)^{n+1} ; \frac{q}{p}\right)_{\infty}}{\left(\left(\frac{q}{p}\right)^{\alpha+n+1} ; \frac{q}{p}\right)_{\infty}} f\left(\left(1-\left(\frac{q}{p}\right)^{n}\right) a+\left(\frac{q}{p}\right)^{n} b\right) \\
& -\sum_{n=0}^{\infty} \frac{\left(\left(\frac{q}{p}\right)^{n+1} ; \frac{q}{p}\right)_{\infty}}{\left(\left(\frac{q}{p}\right)^{\alpha+n+1} ; \frac{q}{p}\right)_{\infty}} f\left(\left(1-\left(\frac{q}{p}\right)^{n+1}\right) a+\left(\frac{q}{p}\right)^{n+1} b\right) \\
& =\sum_{n=0}^{\infty}\left(1-\left(\frac{q}{p}\right)^{\alpha+n}\right) \frac{\left(\left(\frac{q}{p}\right)^{n+1} ; \frac{q}{p}\right)_{\infty}}{\left(\left(\frac{q}{p}\right)^{\alpha+n} ; \frac{q}{p}\right)_{\infty}} f\left(\left(1-\left(\frac{q}{p}\right)^{n}\right) a+\left(\frac{q}{p}\right)^{n} b\right) \\
& -\sum_{n=0}^{\infty}\left(1-\left(\frac{q}{p}\right)^{n+1}\right) \frac{\left(\left(\frac{q}{p}\right)^{n+2} ; \frac{q}{p}\right)_{\infty}}{\left(\left(\frac{q}{p}\right)^{\alpha+n+1} ; \frac{q}{p}\right)_{\infty}} f\left(\left(1-\left(\frac{q}{p}\right)^{n+1}\right) a+\left(\frac{q}{p}\right)^{n+1} b\right) \\
& =\sum_{n=0}^{\infty} \frac{\left(\left(\frac{q}{p}\right)^{n+1} ; \frac{q}{p}\right)_{\infty}}{\left(\left(\frac{q}{p}\right)^{\alpha+n} ; \frac{q}{p}\right)_{\infty}} f\left(\left(1-\left(\frac{q}{p}\right)^{n}\right) a+\left(\frac{q}{p}\right)^{n} b\right) \\
& -\sum_{n=0}^{\infty} \frac{\left(\left(\frac{q}{p}\right)^{n+2} ; \frac{q}{p}\right)_{\infty}}{\left(\left(\frac{q}{p}\right)^{\alpha+n+1} ; \frac{q}{p}\right)_{\infty}} f\left(\left(1-\left(\frac{q}{p}\right)^{n+1}\right) a+\left(\frac{q}{p}\right)^{n+1} b\right) \\
& -\left[\sum_{n=0}^{\infty}\left(\frac{q}{p}\right)^{\alpha+n} \frac{\left(\left(\frac{q}{p}\right)^{n+1} ; \frac{q}{p}\right)_{\infty}}{\left(\left(\frac{q}{p}\right)^{\alpha+n} ; \frac{q}{p}\right)_{\infty}} f\left(\left(1-\left(\frac{q}{p}\right)^{n}\right) a+\left(\frac{q}{p}\right)^{n} b\right)\right. \\
& \left.-\sum_{n=0}^{\infty}\left(\frac{q}{p}\right)^{n+1} \frac{\left(\left(\frac{q}{p}\right)^{n+2} ; \frac{q}{p}\right)_{\infty}}{\left(\left(\frac{q}{p}\right)^{\alpha+n+1} ; \frac{q}{p}\right)_{\infty}} f\left(\left(1-\left(\frac{q}{p}\right)^{n+1}\right) a+\left(\frac{q}{p}\right)^{n+1} b\right)\right] \\
& =\frac{\left(\left(\frac{q}{p}\right)^{1} ; \frac{q}{p}\right)_{\infty}}{\left(\left(\frac{q}{p}\right)^{\alpha} ; \frac{q}{p}\right)_{\infty}} f(b)-f(a)-\left[\sum_{n=0}^{\infty}\left(\frac{q}{p}\right)^{\alpha+n} \frac{\left(\left(\frac{q}{p}\right)^{n+1} ; \frac{q}{p}\right)_{\infty}}{\left(\left(\frac{q}{p}\right)^{\alpha+n} ; \frac{q}{p}\right)_{\infty}} f\left(\left(1-\left(\frac{q}{p}\right)^{n}\right) a+\left(\frac{q}{p}\right)^{n} b\right)\right. \\
& \left.-\sum_{n=1}^{\infty}\left(\frac{q}{p}\right)^{n} \frac{\left(\left(\frac{q}{p}\right)^{n+1} ; \frac{q}{p}\right)_{\infty}}{\left(\left(\frac{q}{p}\right)^{\alpha+n} ; \frac{q}{p}\right)_{\infty}} f\left(\left(1-\left(\frac{q}{p}\right)^{n}\right) a+\left(\frac{q}{p}\right)^{n} b\right)\right] \\
& =\frac{\left(\left(\frac{q}{p}\right)^{1} ; \frac{q}{p}\right)_{\infty}}{\left(\left(\frac{q}{p}\right)^{\alpha} ; \frac{q}{p}\right)_{\infty}} f(b)-f(a)-\left[\sum_{n=0}^{\infty}\left(\frac{q}{p}\right)^{\alpha+n} \frac{\left(\left(\frac{q}{p}\right)^{n+1} ; \frac{q}{p}\right)_{\infty}}{\left(\left(\frac{q}{p}\right)^{\alpha+n} ; \frac{q}{p}\right)_{\infty}} f\left(\left(1-\left(\frac{q}{p}\right)^{n}\right) a+\left(\frac{q}{p}\right)^{n} b\right)\right. \\
& \left.-\sum_{n=0}^{\infty}\left(\frac{q}{p}\right)^{n} \frac{\left(\left(\frac{q}{p}\right)^{n+1} ; \frac{q}{p}\right)_{\infty}}{\left(\left(\frac{q}{p}\right)^{\alpha+n} ; \frac{q}{p}\right)_{\infty}} f\left(\left(1-\left(\frac{q}{p}\right)^{n}\right) a+\left(\frac{q}{p}\right)^{n} b\right)+\frac{\left(\left(\frac{q}{p}\right)^{1} ; \frac{q}{p}\right)_{\infty}}{\left(\left(\frac{q}{p}\right)^{\alpha} ; \frac{q}{p}\right)_{\infty}} f(b)\right] \\
& =-f(a)+\left(1-\left(\frac{q}{p}\right)^{\alpha}\right) \sum_{n=0}^{\infty}\left(\frac{q}{p}\right)^{n} \frac{\left(\left(\frac{q}{p}\right)^{n+1} ; \frac{q}{p}\right)_{\infty}}{\left(\left(\frac{q}{p}\right)^{\alpha+n} ; \frac{q}{p}\right)_{\infty}} f\left(\left(1-\left(\frac{q}{p}\right)^{n}\right) a+\left(\frac{q}{p}\right)^{n} b\right) \\
& =-f(a)+\frac{[\alpha]_{p, q}(p-q)}{p^{\alpha}} \sum_{n=0}^{\infty}\left(\frac{q}{p}\right)^{n} \frac{\left(\left(\frac{q}{p}\right)^{n+1} ; \frac{q}{p}\right)_{\infty}}{\left(\left(\frac{q}{p}\right)^{\alpha+n} ; \frac{q}{p}\right)_{\infty}} f\left(\left(1-\left(\frac{q}{p}\right)^{n}\right) a+\left(\frac{q}{p}\right)^{n} b\right) \\
& =-f(a)+\frac{[\alpha]_{p, q} \Gamma_{p, q}(\alpha)}{p^{\alpha^{2}}(b-a)^{\alpha}} \frac{(p-q) p^{\alpha}(b-a)}{p^{\left(\begin{array}{c}
\alpha \\
2
\end{array}\right)} \Gamma_{p, q}(\alpha)} \sum_{n=0}^{\infty} \frac{q^{n}}{p^{n+1}} p^{\alpha(\alpha-1)}(b-a)^{\alpha-1} p^{\left(\begin{array}{c}
\alpha-1 \\
2
\end{array}\right)} \\
& \times \frac{\left(\left(\frac{q}{p}\right)^{n+1} ; \frac{q}{p}\right)_{\infty}}{\left(\left(\frac{q}{p}\right)^{(\alpha-1)+(n+1)} ; \frac{q}{p}\right)_{\infty}} f\left(\left(1-\left(\frac{q}{p}\right)^{n}\right) a+\left(\frac{q}{p}\right)^{n} b\right) \\
& =-f(a)+\frac{\Gamma_{p, q}(\alpha+1)}{p^{\alpha^{2}}(b-a)^{\alpha}}\left[\frac{1}{p^{\left(\begin{array}{c}
\alpha \\
2
\end{array}\right)} \Gamma_{p, q}(\alpha)} \int_{a}^{a \Phi_{p^{\alpha}}(b)} a\left({ }_{a} \Phi_{p^{\alpha}}(b)-{ }_{a} \Phi_{q}(t)\right)_{p, q}^{(\alpha-1)}\right.
\end{aligned}
$$




$$
\begin{aligned}
& \left.\times f\left(\frac{t}{p^{\alpha-1}}+\left(1-\frac{1}{p^{\alpha-1}}\right) a\right){ }_{a} d_{p, q} t\right] \\
= & -f(a)+\frac{\Gamma_{p, q}(\alpha+1)}{p^{\alpha^{2}}(b-a)^{\alpha}}\left({ }_{a} I_{p, q}^{\alpha} f\right)\left(p^{\alpha} b+\left(1-p^{\alpha}\right) a\right),
\end{aligned}
$$

and

$$
\begin{aligned}
A_{2}= & \frac{p^{\alpha}(b-a)}{[\alpha+1]_{p, q}} \int_{0}^{1}{ }_{a} D_{p, q} f((1-t) a+t b)_{0} d_{p, q} t \\
= & \frac{p^{\alpha}(b-a)}{[\alpha+1]_{p, q}} \int_{0}^{1} \frac{f((1-p t) a+p t b)-f((1-q t) a+q t b)}{(p-q)(b-a) t}{ }_{0} d_{p, q} t \\
= & {\left[\frac{p^{\alpha}}{(p-q)[\alpha+1]_{p, q}} \int_{0}^{1} \frac{f((1-p t) a+p t b)}{t}{ }_{0} d_{p, q} t\right.} \\
& \left.-\frac{p^{\alpha}}{(p-q)[\alpha+1]_{p, q}} \int_{0}^{1} \frac{f((1-q t) a+q t b)}{t}{ }_{0} d_{p, q} t\right] \\
= & \frac{p^{\alpha}}{[\alpha+1]_{p, q}}\left[\sum_{n=0}^{\infty} f\left(\left(1-\left(\frac{q}{p}\right)^{n}\right) a+\left(\frac{q}{p}\right)^{n} b\right)\right. \\
& \left.-\sum_{n=0}^{\infty} f\left(\left(1-\left(\frac{q}{p}\right)^{n+1}\right) a+\left(\frac{q}{p}\right)^{n+1} b\right)\right] \\
= & \frac{p^{\alpha} f(b)-p^{\alpha} f(a)}{[\alpha+1]_{p, q}} .
\end{aligned}
$$

From (3.2) and (3.3), we obtain

$$
\begin{aligned}
& \frac{(b-a)}{[\alpha+1]_{p, q}} \int_{0}^{1}\left(\frac{[\alpha+1]_{p, q}}{p^{\left(\begin{array}{c}
\alpha \\
2
\end{array}\right)}}\left(1-{ }_{0} \Phi_{q}(s)\right)_{p, q}^{(\alpha)}-p^{\alpha}\right){ }_{a} D_{p, q} f((1-t) a+t b){ }_{0} d_{p, q} t \\
& =A_{1}-A_{2} \\
& =\frac{\Gamma_{p, q}(\alpha+1)}{p^{\alpha^{2}}(b-a)^{\alpha}}\left({ }_{a} I_{p, q}^{\alpha} f\right)\left(p^{\alpha} b+\left(1-p^{\alpha}\right) a\right)-\frac{\left([\alpha+1]_{p, q}-p^{\alpha}\right) f(a)+p^{\alpha} f(b)}{[\alpha+1]_{p, q}} .
\end{aligned}
$$

Thus the proof is completed.

Remark 3.1 If $\alpha=1$, then (3.1) reduces to Lemma 3.2 in [43] as

$$
\begin{aligned}
& \frac{1}{p(b-a)} \int_{a}^{p b+(1-p) a} f(x)_{a} d_{p, q} x-\frac{p f(a)+q f(a)}{p+q} \\
& \quad=\frac{q(b-a)}{p+q} \int_{0}^{1}(1-(p+q) t)_{a} D_{p, q} f(t b+(1-t) a)_{a} d_{p, q} t .
\end{aligned}
$$

If $p=1$, then (3.1) reduces to Lemma 5.2 in [40] as

$$
\begin{aligned}
& \frac{\Gamma_{q}(\alpha+1)}{(b-a)^{\alpha}}\left({ }_{a} I_{q}^{\alpha} f\right)(b)-\frac{\left([\alpha+1]_{q}-1\right) f(a)+f(b)}{[\alpha+1]_{q}} \\
& \quad=\frac{(b-a)}{[\alpha+1]_{q}} \int_{0}^{1}\left([\alpha+1]_{q}\left(1-\Phi_{q}(t)\right)_{q}^{(\alpha)}-1\right)_{a} D_{q} f((1-t) a+t b)_{0} d_{q} t .
\end{aligned}
$$


Moreover, if $q \rightarrow 1$ and $\alpha=1$, then (3.6) reduces to

$$
\frac{f(a)+f(b)}{2}-\frac{1}{b-a} \int_{a}^{b} f(x) d x=\frac{b-a}{2} \int_{0}^{1}(1-2 t) f^{\prime}(t a+(1-t) b) d t
$$

which can be found in [76].

Theorem 3.1 Let $f:[a, b] \rightarrow \mathbb{R}$ be a continuous function, $\alpha>0$, and ${ }_{a} D_{p, q} f$ be $(p, q)$ integrable on $\left(a, \frac{1}{p}(b-a)+a\right)$. If $\left|{ }_{a} D_{p, q} f\right|$ is convex on

$$
\left(a, \frac{1}{p}(b-a)+a\right)
$$

then the following Riemann-Liouville fractional $(p, q)$-trapezoid type inequality holds:

$$
\begin{aligned}
& \left|\frac{\Gamma_{p, q}(\alpha+1)}{p^{\alpha^{2}}(b-a)^{\alpha}}\left({ }_{a} I_{p, q}^{\alpha} f\right)\left(p^{\alpha} b+\left(1-p^{\alpha}\right) a\right)-\frac{\left([\alpha+1]_{p, q}-p^{\alpha}\right) f(a)+p^{\alpha} f(b)}{[\alpha+1]_{p, q}}\right| \\
& \quad \leq \frac{(b-a)}{[\alpha+1]_{p, q}}\left(\left|{ }_{a} D_{p, q} f(a)\right| B_{1}+\left|{ }_{a} D_{p, q} f(b)\right| B_{2}\right),
\end{aligned}
$$

where

$$
B_{1}=\int_{0}^{1}\left|\frac{[\alpha+1]_{p, q}}{p^{\left(\begin{array}{c}
\alpha \\
2
\end{array}\right)}}\left(1-{ }_{0} \Phi_{q}(t)\right)_{p, q}^{(\alpha)}-p^{\alpha}\right|(1-t){ }_{0} d_{p, q} t
$$

and

$$
B_{2}=\int_{0}^{1}\left|\frac{[\alpha+1]_{p, q}}{p^{\left(\begin{array}{c}
\alpha \\
2
\end{array}\right)}}\left(1-{ }_{0} \Phi_{q}(t)\right)_{p, q}^{(\alpha)}-p^{\alpha}\right| t_{0} d_{p, q} t
$$

Proof Using Lemma 3.1 and the convexity of $\left|{ }_{a} D_{p, q} f\right|$, we have

$$
\begin{aligned}
\left|\frac{\Gamma_{p, q}(\alpha+1)}{p^{\alpha^{2}}(b-a)^{\alpha}}\left({ }_{a} I_{p, q}^{\alpha} f\right)\left(p^{\alpha} b+\left(1-p^{\alpha}\right) a\right)-\frac{\left([\alpha+1]_{p, q}-p^{\alpha}\right) f(a)+p^{\alpha} f(b)}{[\alpha+1]_{p, q}}\right| \\
\leq \frac{(b-a)}{[\alpha+1]_{p, q}} \int_{0}^{1} \mid \frac{[\alpha+1]_{p, q}}{p^{\left(\begin{array}{c}
\alpha \\
2
\end{array}\right)}\left(1-{ }_{0} \Phi_{q}(t)\right)_{p, q}^{(\alpha)}-\left.p^{\alpha}||{ }_{a} D_{p, q} f((1-t) a+t b)\right|_{0} d_{p, q} t} \\
\leq \frac{(b-a)}{[\alpha+1]_{p, q}} \int_{0}^{1} \mid \frac{[\alpha+1]_{p, q}}{p^{\left(\begin{array}{c}
\alpha \\
2
\end{array}\right)}\left(1-{ }_{0} \Phi_{q}(t)\right)_{p, q}^{(\alpha)}-p^{\alpha} \mid} \\
\quad \times\left[\left|{ }_{a} D_{p, q} f(a)\right|(1-t)+\left|{ }_{a} D_{p, q} f(b)\right| t\right]_{0} d_{p, q} t \\
\leq \frac{(b-a)}{[\alpha+1]_{p, q}}\left[\left|{ }_{a} D_{p, q} f(a)\right| \int_{0}^{1} \mid \frac{[\alpha+1]_{p, q}}{\left.p^{\left(\begin{array}{c}
\alpha \\
2
\end{array}\right)}\left(1-{ }_{0} \Phi_{q}(t)\right)_{p, q}^{(\alpha)}-p^{\alpha} \mid(1-t)\right]{ }_{0} d_{p, q} t}\right. \\
\quad+\frac{(b-a)}{[\alpha+1]_{p, q}}\left[\left|{ }_{a} D_{p, q} f(b)\right| \int_{0}^{1} \mid \frac{[\alpha+1]_{p, q}}{\left.p^{\left(\begin{array}{c}
\alpha \\
2
\end{array}\right)}\left(1-{ }_{0} \Phi_{q}(t)\right)_{p, q}^{(\alpha)}-p^{\alpha} \mid t\right]{ }_{0} d_{p, q} t .}\right.
\end{aligned}
$$

This completes the proof. 
Remark 3.2 If $p=1$, then (3.8) reduces to

$$
\begin{gathered}
\left|\frac{\Gamma_{q}(\alpha+1)}{(b-a)^{\alpha}}\left({ }_{a} I_{q}^{\alpha} f\right)(b)-\frac{\left([\alpha+1]_{q}-1\right) f(a)+f(b)}{[\alpha+1]_{q}}\right| \\
\quad \leq \frac{(b-a)}{[\alpha+1]_{q}}\left(\left|{ }_{a} D_{q} f(a)\right| \delta_{1}+\left|{ }_{a} D_{q} f(b)\right| \delta_{2}\right),
\end{gathered}
$$

where

$$
\delta_{1}=\int_{0}^{1}\left|[\alpha+1]_{q}\left(1-{ }_{0} \Phi_{q}(t)\right)_{q}^{(\alpha)}-1\right|(1-t)_{0} d_{q} t
$$

and

$$
\delta_{2}=\int_{0}^{1}\left|[\alpha+1]_{q}\left(1-{ }_{0} \Phi_{q}(t)\right)_{q}^{(\alpha)}-1\right| t_{0} d_{q} t
$$

which appeared in [40].

Theorem 3.2 Let $f:[a, b] \rightarrow \mathbb{R}$ be a continuous function, $\alpha>0$, and ${ }_{a} D_{p, q} f$ be $(p, q)$ integrable on $\left(a, \frac{1}{p}(b-a)+a\right)$. If $\left|{ }_{a} D_{p, q} f\right|^{r}$ is convex on $\left(a, \frac{1}{p}(b-a)+a\right)$ for $r \geq 0$, then the following Riemann-Liouville fractional $(p, q)$-trapezoid type inequality holds:

$$
\begin{aligned}
& \left|\frac{\Gamma_{p, q}(\alpha+1)}{p^{\alpha^{2}}(b-a)^{\alpha}}\left({ }_{a} I_{p, q}^{\alpha} f\right)\left(p^{\alpha} b+\left(1-p^{\alpha}\right) a\right)-\frac{\left([\alpha+1]_{p, q}-p^{\alpha}\right) f(a)+p^{\alpha} f(b)}{[\alpha+1]_{p, q}}\right| \\
& \quad \leq \frac{(b-a)}{[\alpha+1]_{p, q}} B_{3}^{1-1 / r}\left(\left|{ }_{a} D_{p, q} f(a)\right|^{r} B_{1}+\left|{ }_{a} D_{p, q} f(b)\right|^{r} B_{2}\right)^{1 / r}
\end{aligned}
$$

where $B_{1}$ and $B_{2}$ are given in Theorem 3.1 and

$$
B_{3}=\int_{0}^{1}\left|\frac{[\alpha+1]_{p, q}}{p^{\left(\begin{array}{c}
\alpha \\
2
\end{array}\right)}}\left(1-{ }_{0} \Phi_{q}(t)\right)_{p, q}^{(\alpha)}-p^{\alpha}\right|{ }_{0} d_{p, q} t .
$$

Proof Using Lemma 3.1, the convexity of $\left.\left.\right|_{a} D_{p, q} f\right|^{r}$, and the power mean inequality, we have

$$
\begin{aligned}
& \left|\frac{\Gamma_{p, q}(\alpha+1)}{p^{\alpha^{2}}(b-a)^{\alpha}}\left({ }_{a} I_{p, q}^{\alpha} f\right)\left(p^{\alpha} b+\left(1-p^{\alpha}\right) a\right)-\frac{\left([\alpha+1]_{p, q}-p^{\alpha}\right) f(a)+p^{\alpha} f(b)}{[\alpha+1]_{p, q}}\right|
\end{aligned}
$$

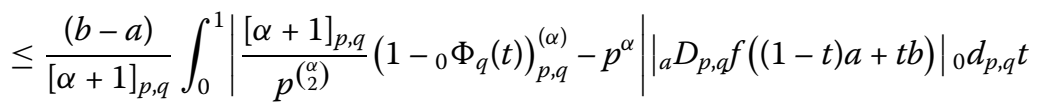

$$
\begin{aligned}
& \leq \frac{(b-a)}{[\alpha+1]_{p, q}}\left(\int_{0}^{1}\left|\frac{[\alpha+1]_{p, q}}{p^{\left(\begin{array}{c}
\alpha \\
2
\end{array}\right)}}\left(1-{ }_{0} \Phi_{q}(t)\right)_{p, q}^{(\alpha)}-p^{\alpha}\right|{ }_{0} d_{p, q} t\right)^{1-1 / r} \\
& \times\left(\left.\int_{0}^{1}\left|\frac{[\alpha+1]_{p, q}}{p^{\left(\begin{array}{c}
\alpha \\
2
\end{array}\right)}}\left(1-{ }_{0} \Phi_{q}(t)\right)_{p, q}^{(\alpha)}-p^{\alpha}\right|{ }_{a} D_{p, q} f((1-t) a+t b)\right|_{0} ^{r} d_{p, q} t\right)^{1 / r} \\
& \leq \frac{(b-a)}{[\alpha+1]_{p, q}}\left(\int_{0}^{1}\left|\frac{[\alpha+1]_{p, q}}{p^{\left(\begin{array}{c}
\alpha \\
2
\end{array}\right)}}\left(1-{ }_{0} \Phi_{q}(t)\right)_{p, q}^{(\alpha)}-p^{\alpha}\right|{ }_{0} d_{p, q} t\right)^{1-1 / r} \\
& \times\left(\int _ { 0 } ^ { 1 } | \frac { [ \alpha + 1 ] _ { p , q } } { p ^ { ( \begin{array} { c } 
{ \alpha } \\
{ 2 }
\end{array} ) } } ( 1 - { } _ { 0 } \Phi _ { q } ( t ) ) _ { p , q } ^ { ( \alpha ) } - p ^ { \alpha } | \left[\left|{ }_{a} D_{p, q} f(a)\right|^{r}(1-t)\right.\right.
\end{aligned}
$$




$$
\begin{aligned}
& \left.\left.+\left|{ }_{a} D_{p, q} f(b)\right|^{r} t\right]_{0} d_{p, q} t\right)^{1 / r} \\
& \leq \frac{(b-a)}{[\alpha+1]_{p, q}}\left(\int_{0}^{1}\left|\frac{[\alpha+1]_{p, q}}{p^{(\alpha)}\left(\begin{array}{c}
\alpha \\
2
\end{array}\right.}\left(1-{ }_{0} \Phi_{q}(t)\right)_{p, q}^{(\alpha)}-p^{\alpha}\right|{ }_{0} d_{p, q} t\right)^{1-1 / r} \\
& \times\left[\left|{ }_{a} D_{p, q} f(a)\right|^{r} \int_{0}^{1}\left|\frac{[\alpha+1]_{p, q}}{p^{\left(\begin{array}{c}
\alpha \\
2
\end{array}\right)}}\left(1-{ }_{0} \Phi_{q}(t)\right)_{p, q}^{(\alpha)}-p^{\alpha}\right|(1-t)_{0} d_{p, q} t\right. \\
& \left.+\left|{ }_{a} D_{p, q} f(b)\right|^{r} \int_{0}^{1}\left|\frac{[\alpha+1]_{p, q}}{p^{\left(\begin{array}{c}
\alpha \\
2
\end{array}\right)}}\left(1-{ }_{0} \Phi_{q}(t)\right)_{p, q}^{(\alpha)}-p^{\alpha}\right|(1-t)_{0} d_{p, q} t\right]^{1 / r} .
\end{aligned}
$$

Therefore, the proof is completed.

Remark 3.3 If $\alpha=1$, then (3.10) reduces to

$$
\begin{aligned}
& \left|\frac{1}{p(b-a)} \int_{a}^{p b+(1-p) a} f(x){ }_{a} d_{p, q} x-\frac{p f(a)+q f(a)}{p+q}\right| \\
& \quad=\frac{q(b-a)}{p+q}\left[\frac{2(p+q-1)}{(p+q)^{2}}\right]^{1-1 / r}\left[\lambda_{1}(p, q)\left|{ }_{a} D_{p, q} f(b)\right|^{r}+\lambda_{2}(p, q)\left|{ }_{a} D_{p, q} f(a)\right|^{r}\right]^{1 / r},
\end{aligned}
$$

where

$$
\lambda_{1}(p, q)=\frac{q\left[\left(p^{3}-2+2 p\right)+\left(2 p^{2}+2\right) q+p q^{2} p q^{2}\right]+2 p^{2}-2 p}{(p+q)^{3}\left(p^{2}+p q+q^{2}\right)}
$$

and

$$
\begin{aligned}
\lambda_{2}(p, q)= & \frac{1}{(p+q)^{3}\left(p^{2}+p q+q^{2}\right)}\left\{q \left[\left(5 p^{3}-4 p^{2}-2 p+2\right)+\left(6 p^{2}-4 p-2\right) q\right.\right. \\
& \left.\left.+(5 p-2) q^{2}+2 q^{3}\right]+\left(2 p^{4}-2 p^{3}-2 p^{3}-2 p^{2}+2 p\right)\right\},
\end{aligned}
$$

which appeared in [43].

Moreover, if $p=1$, then (3.10) reduces to

$$
\begin{aligned}
& \left|\frac{\Gamma_{q}(\alpha+1)}{(b-a)^{\alpha}}\left({ }_{a} I_{q}^{\alpha} f\right)(b)-\frac{\left([\alpha+1]_{q}-1\right) f(a)+f(b)}{[\alpha+1]_{q}}\right| \\
& \quad \leq \frac{(b-a)}{[\alpha+1]_{q}} M_{3}^{1-1 / r}\left(\left.\left.\right|_{a} D_{q} f(a)\right|^{r} M_{1}+\left.\left.\right|_{a} D_{q} f(b)\right|^{r} M_{2}\right)^{1 / r},
\end{aligned}
$$

where $\delta_{1}$ and $\delta_{2}$ are given in Remark 3.2 and

$$
\delta_{3}=\int_{0}^{1}\left|[\alpha+1]_{q}\left(1-{ }_{0} \Phi_{q}(t)\right)_{q}^{(\alpha)}-1\right|{ }_{0} d_{q} t
$$

which appeared in [40].

Theorem 3.3 Let $f:[a, b] \rightarrow \mathbb{R}$ be a continuous function, $\alpha>0$ and ${ }_{a} D_{p, q} f$ be $(p, q)$ integrable on $\left(a, \frac{1}{p}(b-a)+a\right)$. If $\left|{ }_{a} D_{p, q} f\right|^{r}$ is convex on $\left[a, \frac{1}{p}(b-a)+a\right]$ for $r>1$ and 
$1 / r+1 / p=1$, then the following Riemann-Liouville fractional $(p, q)$-trapezoid type inequality holds:

$$
\begin{aligned}
& \left|\frac{\Gamma_{p, q}(\alpha+1)}{p^{\alpha^{2}}(b-a)^{\alpha}}\left({ }_{a} I_{p, q}^{\alpha} f\right)\left(p^{\alpha} b+\left(1-p^{\alpha}\right) a\right)-\frac{\left([\alpha+1]_{p, q}-p^{\alpha}\right) f(a)+p^{\alpha} f(b)}{[\alpha+1]_{p, q}}\right| \\
& \quad \leq \frac{(b-a)}{[\alpha+1]_{p, q}} B_{4}^{1 / s}\left(\frac{\left.\left.(p+q-1)\right|_{a} D_{p, q} f(a)\right|^{r}+\left.\left.\right|_{a} D_{p, q} f(b)\right|^{r}}{p+q}\right)^{1 / r}
\end{aligned}
$$

where

$$
B_{4}=\int_{0}^{1}\left|\frac{[\alpha+1]_{p, q}}{p^{\left(\begin{array}{c}
\alpha \\
2
\end{array}\right)}}\left(1-{ }_{0} \Phi_{q}(t)\right)_{p, q}^{(\alpha)}-p^{\alpha}\right|^{s}{ }_{0} d_{p, q} t .
$$

Proof Using Lemma 3.1, the convexity of $\left|{ }_{a} D_{p, q} f\right|^{r}$, and Hölder's inequality, we have

$$
\begin{aligned}
\left|\frac{\Gamma_{p, q}(\alpha+1)}{p^{\alpha^{2}}(b-a)^{\alpha}}\left({ }_{a} I_{p, q}^{\alpha} f\right)\left(p^{\alpha} b+\left(1-p^{\alpha}\right) a\right)-\frac{\left([\alpha+1]_{p, q}-p^{\alpha}\right) f(a)+p^{\alpha} f(b)}{[\alpha+1]_{p, q}}\right| \\
\leq\left.\frac{(b-a)}{[\alpha+1]_{p, q}} \int_{0}^{1}\left|\frac{[\alpha+1]_{p, q}}{p^{\left(\begin{array}{c}
\alpha \\
2
\end{array}\right)}\left(1-{ }_{0} \Phi_{q}(t)\right)_{p, q}^{(\alpha)}-p^{\alpha} \mid}\right|{ }_{a} D_{p, q} f((1-t) a+t b)\right|_{0} d_{p, q} t \\
\leq \frac{(b-a)}{[\alpha+1]_{p, q}}\left(\int_{0}^{1} \mid \frac{[\alpha+1]_{p, q}}{\left.p^{\left(\begin{array}{c}
\alpha \\
2
\end{array}\right)}\left(1-{ }_{0} \Phi_{q}(t)\right)_{p, q}^{(\alpha)}-\left.p^{\alpha}\right|^{s}{ }_{0} d_{p, q} t\right)^{1 / s}}\right. \\
\quad \times\left(\int_{0}^{1}\left|{ }_{a} D_{p, q} f((1-t) a+t b)\right|^{r}{ }_{0} d_{p, q} t\right){ }^{1 / r} \\
\leq \frac{(b-a)}{[\alpha+1]_{p, q}}\left(\int_{0}^{1} \mid \frac{[\alpha+1]_{p, q}}{\left.p^{\left(\begin{array}{c}
\alpha \\
2
\end{array}\right)}\left(1-{ }_{0} \Phi_{q}(t)\right)_{p, q}^{(\alpha)}-\left.p^{\alpha}\right|^{s}{ }_{0} d_{p, q} t\right)^{1 / s}}\right. \\
\quad \times\left(\int_{0}^{1}\left[\left.\left.\right|_{a} D_{p, q} f(a)\right|^{r}(1-t)+\left.{ }_{a} D_{p, q} f(b)\right|^{r} t\right]_{0} d_{p, q} t\right)^{1 / r} \\
\leq \frac{(b-a)}{[\alpha+1]_{p, q}}\left(\int_{0}^{1} \mid \frac{[\alpha+1]_{p, q}}{\left.p^{\left(\begin{array}{c}
\alpha \\
2
\end{array}\right)}\left(1-{ }_{0} \Phi_{q}(t)\right)_{p, q}^{(\alpha)}-\left.p^{\alpha}\right|^{s}{ }_{0} d_{p, q} t\right)^{1 / s}}\right. \\
\quad \times\left(\frac{\left.\left.(p+q-1)\right|_{a} D_{p, q} f(a)\right|^{r}+\left.{ }_{a} D_{p, q} f(b)\right|^{r}}{p+q}\right)^{1 / r} \cdot
\end{aligned}
$$

This completes the proof.

Remark 3.4 If $\alpha=1$, then (3.13) reduces to

$$
\begin{aligned}
& \left|\frac{1}{p(b-a)} \int_{a}^{p b+(1-p) a} f(x)_{a} d_{p, q} x-\frac{p f(a)+q f(a)}{p+q}\right| \\
& \quad=\frac{q(b-a)}{p+q}\left[\lambda_{3}\right]^{1 / s}\left(\frac{\left|{ }_{a} D_{p, q} f(b)\right|^{r}+\left.\left.(p+q-1)\right|_{a} D_{p, q} f(a)\right|^{r}}{p+q}\right)^{1 / r},
\end{aligned}
$$

where

$$
\lambda_{3}=\int_{0}^{1}|1-(p+q) t|_{0}^{s} d_{p, q} t
$$

which appeared in [43]. 
Moreover, if $p=1$, then (3.13) reduces to

$$
\begin{gathered}
\left|\frac{\Gamma_{q}(\alpha+1)}{(b-a)^{\alpha}}\left({ }_{a} I_{q}^{\alpha} f\right)(b)-\frac{\left([\alpha+1]_{q}-\right) f(a)+f(b)}{[\alpha+1]_{q}}\right| \\
\quad \leq \frac{(b-a)}{[\alpha+1]_{q}} \delta_{4}^{1 / s}\left(\frac{\left.\left.q\right|_{a} D_{q} f(a)\right|^{r}+\left|{ }_{a} D_{q} f(b)\right|^{r}}{1+q}\right)^{1 / r},
\end{gathered}
$$

where

$$
\delta_{4}=\int_{0}^{1}\left|[\alpha+1]_{q}\left(1-{ }_{0} \Phi_{q}(t)\right)_{q}^{(\alpha)}-1\right|^{s}{ }_{0} d_{q} t
$$

which appeared in [40].

Now we will prove the following lemma to obtain the Riemann-Liouville fractional $(p, q)$-midpoint type inequalities.

Lemma 3.2 Let $f:[a, b] \rightarrow \mathbb{R}$ be a continuous function and $\alpha>0$. If ${ }_{a} D_{p, q} f$ is $(p, q)$ integrable on $\left(a, \frac{1}{p}(b-a)+a\right)$, then the following equality holds:

$$
\begin{gathered}
f\left(\frac{\left([\alpha+1]_{p, q}-p^{\alpha}\right) a+p^{\alpha} b}{[\alpha+1]_{p, q}}\right)-\frac{\Gamma_{p, q}(\alpha+1)}{p^{\alpha^{2}}(b-a)^{\alpha}}\left({ }_{a} I_{p, q}^{\alpha} f\right)\left(p^{\alpha} b+\left(1-p^{\alpha}\right) a\right) \\
=(b-a)\left[\int_{0}^{\frac{p^{\alpha}}{[\alpha+1]_{p, q}}}\left(1-\frac{\left(1-{ }_{0} \Phi_{q}(t)\right)_{p, q}^{(\alpha)}}{p^{\left(\begin{array}{c}
\alpha \\
2
\end{array}\right)}}\right){ }_{a} D_{p, q} f((1-t) a+t b)_{0} d_{p, q} t\right. \\
\left.\quad+\int_{\frac{p^{\alpha}}{[\alpha+1]_{p, q}}}^{1}-\frac{\left(1-{ }_{0} \Phi_{q}(t)\right)_{p, q}^{(\alpha)}}{p^{\left(\begin{array}{c}
\alpha \\
2
\end{array}\right)}}{ }_{a} D_{p, q} f((1-t) a+t b)_{0} d_{p, q} t\right] .
\end{gathered}
$$

Proof By direct computation and using Definitions 2.1 and 2.2, we have

$$
\begin{aligned}
& A_{3}=\int_{0}^{\frac{p^{\alpha}}{[\alpha+1]_{p, q}}}{ }_{a} D_{p, q} f((1-t) a+t b){ }_{0} d_{p, q} t \\
& =\int_{0}^{\frac{p^{\alpha}}{[\alpha+1]_{p, q}}} \frac{f((1-p t) a+p t b)-f((1-q t) a+q t b)}{(p-q)(b-a) t}{ }_{0} d_{p, q} t \\
& =\frac{1}{(p-q)(b-a)} \int_{0}^{\frac{p^{\alpha}}{[\alpha+1]_{p, q}}} \frac{f((1-p t) a+p t b)}{t}{ }_{0} d_{p, q} t \\
& -\frac{1}{(p-q)(b-a)} \int_{0}^{\frac{p^{\alpha}}{[\alpha+1]_{p, q}}} \frac{f((1-q t) a+q t b)}{t}{ }_{0} d_{p, q} t \\
& =\frac{p^{\alpha}}{(b-a)[\alpha+1]_{p, q}} \sum_{n=0}^{\infty} \frac{q^{n}}{p^{n+1}} \frac{f\left(\left(1-\frac{q^{n} p^{\alpha}}{p^{n}[\alpha+1]_{p, q}}\right) a+\frac{q^{n} p^{\alpha}}{p^{n}[\alpha+1]_{p, q}} b\right)}{\frac{q^{n} p^{\alpha}}{p^{n+1}[\alpha+1]_{p, q}}} \\
& -\frac{p^{\alpha}}{(b-a)[\alpha+1]_{p, q}} \sum_{n=0}^{\infty} \frac{q^{n}}{p^{n+1}} \frac{f\left(\left(1-\frac{q^{n+1} p^{\alpha}}{p^{n+1}[\alpha+1]_{p, q}}\right) a+\frac{q^{n+1} p^{\alpha}}{p^{n+1}[\alpha+1]_{p, q}} b\right)}{\frac{q^{n} p^{\alpha}}{p^{n+1}[\alpha+1]_{p, q}}}
\end{aligned}
$$




$$
\begin{aligned}
= & \frac{1}{(b-a)}\left[\sum_{n=0}^{\infty} f\left(\left(1-\frac{q^{n} p^{\alpha}}{p^{n}[\alpha+1]_{p, q}}\right) a+\frac{q^{n} p^{\alpha}}{p^{n}[\alpha+1]_{p, q}} b\right)\right. \\
& \left.-\sum_{n=0}^{\infty} f\left(\left(1-\frac{q^{n+1} p^{\alpha}}{p^{n+1}[\alpha+1]_{p, q}}\right) a+\frac{q^{n+1} p^{\alpha}}{p^{n+1}[\alpha+1]_{p, q}} b\right)\right] \\
= & \frac{1}{(b-a)}\left[f\left(\frac{\left([\alpha+1]_{p, q}-p^{\alpha}\right) a+p^{\alpha} b}{[\alpha+1]_{p, q}}\right)-f(a)\right] .
\end{aligned}
$$

On the other hand, in Lemma 3.1, the following integral was given:

$$
\begin{aligned}
A_{1} & =\frac{b-a}{p^{\left(\begin{array}{c}
\alpha \\
2
\end{array}\right)}} \int_{0}^{1}\left(1-{ }_{0} \Phi_{q}(t)\right)_{p, q}^{(\alpha)}{ }_{a} D_{p, q} f((1-t) a+t b){ }_{0} d_{p, q} t \\
& =-f(a)+\frac{\Gamma_{p, q}(\alpha+1)}{p^{\alpha^{2}}(b-a)^{\alpha}}\left(a_{p, q}^{\alpha} f\right)\left(p^{\alpha} b+\left(1-p^{\alpha}\right) a\right) .
\end{aligned}
$$

Consequently, from (3.17) and (3.18), we have

$$
\begin{aligned}
A_{3}+ & A_{1} \\
= & (b-a)\left[\int_{0}^{\frac{p^{\alpha}}{\alpha+1 p_{p, q}}}\left(1-\frac{\left(1-{ }_{0} \Phi_{q}(t)\right)_{p, q}^{(\alpha)}}{p^{\left(\frac{\alpha}{2}\right)}}\right){ }_{a} D_{p, q} f((1-t) a+t b)_{0} d_{p, q} t\right. \\
& \left.+\int_{\frac{p^{\alpha}}{[\alpha+1]_{p, q}}}^{1}-\frac{\left(1-{ }_{0} \Phi_{q}(t)\right)_{p, q}^{(\alpha)}}{\left.p^{(\alpha)}{ }_{2}\right)}{ }_{a} D_{p, q} f((1-t) a+t b){ }_{0} d_{p, q} t\right] \\
= & (b-a)\left[\int_{0}^{\frac{p^{\alpha}}{\alpha+1] p, q}}{ }_{a} D_{p, q} f((1-t) a+t b)_{0} d_{p, q} t\right. \\
& \left.-\int_{0}^{1} \frac{\left(1-{ }_{0} \Phi_{q}(t)\right)_{p, q}^{(\alpha)}}{p^{\left(\frac{\alpha}{2}\right)}}{ }_{a} D_{p, q} f((1-t) a+t b)_{0} d_{p, q} t\right] \\
= & f\left(\frac{\left([\alpha+1]_{p, q}-p^{\alpha}\right) a+p^{\alpha} b}{[\alpha+1]_{p, q}}\right)-\frac{\Gamma_{p, q}(\alpha+1)}{p^{\alpha^{2}}(b-a)^{\alpha}}\left({ }_{a} I_{p, q}^{\alpha} f\right)\left(p^{\alpha} b+\left(1-p^{\alpha}\right) a\right) .
\end{aligned}
$$

Therefore, the proof is completed.

Remark 3.5 If $\alpha=1$, then (3.16) reduces to

$$
\begin{aligned}
\left|f\left(\frac{q a+p b}{p+q}\right)-\frac{1}{p(b-a)} \int_{a}^{p b+(1-p) a} f(x){ }_{a} d_{p, q} x\right| \\
=q(b-a)\left[\int_{0}^{\frac{p}{p+q}} t_{a} D_{p, q} f((1-t) a+t b)_{0} d_{p, q} t\right. \\
\left.\quad+\int_{\frac{p}{p+q}}^{1}\left(t-\frac{1}{q}\right){ }_{a} D_{p, q} f((1-t) a+t b)_{0} d_{p, q} t\right],
\end{aligned}
$$

which appeared in [42]. 
Moreover, if $p=1$, then (3.16) reduces to

$$
\begin{aligned}
f( & \left.\frac{\left([\alpha+1]_{q}-1\right) a+b}{[\alpha+1]_{q}}\right)-\frac{\Gamma_{q}(\alpha+1)}{(b-a)^{\alpha}}\left({ }_{a} I_{q}^{\alpha} f\right)(b) \\
= & (b-a)\left[\int_{0}^{\frac{1}{[\alpha+1]_{q}}}\left(1-{ }_{0} \Phi_{q}(t)\right)_{q}^{(\alpha)}{ }_{a} D_{q} f((1-t) a+t b){ }_{0} d_{q} t\right. \\
& \left.-\int_{\frac{1}{[\alpha+1]_{q}}}^{1}\left(1-{ }_{0} \Phi_{q}(t)\right)_{q}^{(\alpha)}{ }_{a} D_{q} f((1-t) a+t b){ }_{0} d_{q} t,\right]
\end{aligned}
$$

which appeared in [40].

Theorem 3.4 Let $f:[a, b] \rightarrow \mathbb{R}$ be a continuous function, $\alpha>0$, and ${ }_{a} D_{p, q} f$ be $(p, q)$ integrable on $\left(a, \frac{1}{p}(b-a)+a\right)$. If $\left.\right|_{a} D_{p, q} f \mid$ is convex on $\left(a, \frac{1}{p}(b-a)+a\right)$, then the following Riemann-Liouville fractional $(p, q)$-midpoint type inequality holds:

$$
\begin{aligned}
& \left|f\left(\frac{\left([\alpha+1]_{p, q}-p^{\alpha}\right) a+p^{\alpha} b}{[\alpha+1]_{p, q}}\right)-\frac{\Gamma_{p, q}(\alpha+1)}{p^{\alpha^{2}}(b-a)^{\alpha}}\left({ }_{a} I_{p, q}^{\alpha} f\right)\left(p^{\alpha} b+\left(1-p^{\alpha}\right) a\right)\right| \\
& \quad \leq(b-a)\left[B_{5}\left|{ }_{a} D_{p, q} f(a)\right|+B_{6}\left|{ }_{a} D_{p, q} f(b)\right|+B_{7}\left|{ }_{a} D_{p, q} f(a)\right|+\left.B_{8}\right|_{a} D_{p, q} f(b) \mid\right]
\end{aligned}
$$

where

$$
\begin{aligned}
& B_{5}=\left[\int_{0}^{\frac{p^{\alpha}}{[\alpha+1]_{p, q}}}\left|1-\frac{\left(1-{ }_{0} \Phi_{q}(t)\right)_{p, q}^{(\alpha)}}{\left.p^{(\alpha} \begin{array}{c}
\alpha \\
2
\end{array}\right)}\right|(1-t)_{0} d_{p, q} t\right], \\
& B_{6}=\left[\int_{0}^{\frac{p^{\alpha}}{[\alpha+1]_{p, q}}}\left|1-\frac{\left(1-{ }_{0} \Phi_{q}(t)\right)_{p, q}^{(\alpha)}}{p^{\left(\begin{array}{c}
\alpha \\
2
\end{array}\right)}}\right| t_{0} d_{p, q} t\right], \\
& B_{7}=\left[\int_{\frac{p^{\alpha}}{[\alpha+1]_{p, q}}}^{1} \mid-\frac{\left(1-{ }_{0} \Phi_{q}(t)\right)_{p, q}^{(\alpha)}}{\left.p^{\left(\begin{array}{c}
\alpha \\
2
\end{array}\right)} \mid(1-t)_{0} d_{p, q} t\right],}\right. \\
& B_{8}=\left[\int_{\frac{p^{\alpha}}{[\alpha+1]_{p, q}}}^{1}\left|-\frac{\left(1-{ }_{0} \Phi_{q}(t)\right)_{p, q}^{(\alpha)}}{p^{\left(\begin{array}{c}
\alpha \\
2
\end{array}\right)}}\right| t_{0} d_{p, q} t\right] .
\end{aligned}
$$

Proof Using Lemma 3.2 and the convexity of $\left|{ }_{a} D_{p, q} f\right|$, we have

$$
\begin{aligned}
& \left|f\left(\frac{\left([\alpha+1]_{p, q}-p^{\alpha}\right) a+p^{\alpha} b}{[\alpha+1]_{p, q}}\right)-\frac{\Gamma_{p, q}(\alpha+1)}{p^{\alpha^{2}}(b-a)^{\alpha}}\left({ }_{a} I_{p, q}^{\alpha} f\right)\left(p^{\alpha} b+\left(1-p^{\alpha}\right) a\right)\right| \\
& \leq(b-a)\left[\int_{0}^{\frac{p^{\alpha}}{[\alpha+1] p, q}}\left|1-\frac{\left(1-{ }_{0} \Phi_{q}(t)\right)_{p, q}^{(\alpha)}}{p^{\left(\begin{array}{c}
\alpha \\
2
\end{array}\right)}}\right|\left|{ }_{a} D_{p, q} f((1-t) a+t b)\right|_{0} d_{p, q} t\right. \\
& \left.+\int_{\frac{p^{\alpha}}{[\alpha+1]_{p, q}}}^{1}\left|-\frac{\left(1-{ }_{0} \Phi_{q}(t)\right)_{p, q}^{(\alpha)}}{p^{\left(\begin{array}{c}
\alpha \\
2
\end{array}\right)}}\right|\left|{ }_{a} D_{p, q} f((1-t) a+t b)\right|_{0} d_{p, q} t\right] \\
& \leq(b-a)\left[\int_{0}^{\frac{p^{\alpha}}{[\alpha+1]_{p, q}}}\left|1-\frac{\left(1-{ }_{0} \Phi_{q}(t)\right)_{p, q}^{(\alpha)}}{p^{\left(\begin{array}{c}
\alpha \\
2
\end{array}\right)}}\right|\left[\left|{ }_{a} D_{p, q} f(a)\right|(1-t)+\left|{ }_{a} D_{p, q} f(b)\right| t\right]_{0} d_{p, q} t\right. \\
& \left.+\int_{\frac{p^{\alpha}}{[\alpha+1]_{p, q}}}^{1}\left|-\frac{\left(1-{ }_{0} \Phi_{q}(t)\right)_{p, q}^{(\alpha)}}{p^{(\alpha)}\left(\begin{array}{l}
\alpha \\
2
\end{array}\right.}\right|\left[\left|{ }_{a} D_{p, q} f(a)\right|(1-t)+\left|{ }_{a} D_{p, q} f(b)\right| t\right]_{0} d_{p, q} t\right]
\end{aligned}
$$




$$
\begin{aligned}
\leq & (b-a)\left[\int_{0}^{\frac{p^{\alpha}}{[\alpha+1] p, q}}\left|1-\frac{\left(1-{ }_{0} \Phi_{q}(t)\right)_{p, q}^{(\alpha)}}{p^{\left(\begin{array}{c}
\alpha \\
2
\end{array}\right)}}\right|{ }_{a} D_{p, q} f(a) \mid(1-t)_{0} d_{p, q} t\right. \\
& \left.+\int_{0}^{\frac{p^{\alpha}}{[\alpha+1] p, q}}\left|1-\frac{\left(1-{ }_{0} \Phi_{q}(t)\right)_{p, q}^{(\alpha)}}{p^{\left(\begin{array}{c}
\alpha \\
2
\end{array}\right)}}\right|{ }_{a} D_{p, q} f(b) \mid t_{0} d_{p, q} t\right] \\
& +(b-a)\left[\int_{\frac{p^{\alpha}}{[\alpha+1] p, q}}^{1}\left|-\frac{\left(1-{ }_{0} \Phi_{q}(t)\right)_{p, q}^{(\alpha)}}{p^{\left(\begin{array}{c}
\alpha \\
2
\end{array}\right)}}\right|{ }_{a} D_{p, q} f(a) \mid(1-t)_{0} d_{p, q} t\right. \\
& \left.+\left.\int_{\frac{p^{\alpha}}{[\alpha+1]_{p, q}}}^{1}\left|-\frac{\left(1-{ }_{0} \Phi_{q}(t)\right)_{)_{p, q}}^{(\alpha)}}{p^{\left(\begin{array}{c}
\alpha \\
2
\end{array}\right)}}\right|\right|_{a} D_{p, q} f(b) \mid t_{0} d_{p, q} t\right] .
\end{aligned}
$$

This completes the proof.

Remark 3.6 If $\alpha=1$, then (3.21) reduces to

$$
\begin{aligned}
&\left.\mid f\left(\frac{q a+p b}{p+q}\right)-\frac{1}{p(b-a)} \int_{a}^{p b+(1-p) a} f(x)\right)_{a} d_{p, q} x \mid \\
& \leq q(b-a)\left[\left.\lambda_{4}(p, q)\right|_{a} D_{p, q} f(a)\left|+\lambda_{5}(p, q)\right|_{a} D_{p, q} f(b) \mid\right. \\
&\left.+\lambda_{6}(p, q)\left|{ }_{a} D_{p, q} f(a)\right|+\left.\lambda_{7}(p, q)\right|_{a} D_{p, q} f(b) \mid\right],
\end{aligned}
$$

where

$$
\begin{array}{ll}
\lambda_{4}(p, q)=\frac{p^{3}}{(p+q)^{3}\left(p^{2}+p q+q^{2}\right)}, & \lambda_{5}(p, q)=\frac{p^{2}\left(p^{2}+p q+q^{2}\right)-p^{3}}{(p+q)^{3}\left(p^{2}+p q+q^{2}\right)} \\
\lambda_{6}(p, q)=\frac{2 p^{3}}{(p+q)^{3}\left(p^{2}+p q+q^{2}\right)}, & \lambda_{7}(p, q)=\frac{p^{4}+p^{3} q+p^{2} q^{2}-2 p^{3}}{(p+q)^{3}\left(p^{2}+p q+q^{2}\right)}
\end{array}
$$

which appeared in [42].

Moreover, if $p=1$, then (3.21) reduces to

$$
\begin{aligned}
& \left|f\left(\frac{\left([\alpha+1]_{q}-1\right) a+b}{[\alpha+1]_{q}}\right)-\frac{\Gamma_{q}(\alpha+1)}{(b-a)^{\alpha}}\left(a I_{q}^{\alpha} f\right)(b)\right| \\
& \quad \leq(b-a)\left[\delta_{5}\left|{ }_{a} D_{q} f(a)\right|+\delta_{6}\left|{ }_{a} D_{q} f(b)\right|+\delta_{7}\left|{ }_{a} D_{q} f(a)\right|+\delta_{8}\left|{ }_{a} D_{q} f(b)\right|\right],
\end{aligned}
$$

where

$$
\begin{aligned}
& \delta_{5}=\left[\int_{0}^{\frac{1}{[\alpha+1] q}}\left|1-\left(1-{ }_{0} \Phi_{q}(t)\right)_{q}^{(\alpha)}\right|(1-t){ }_{0} d_{q} t\right] \\
& \delta_{6}=\left[\int_{0}^{\frac{1}{[\alpha+1]_{q}}}\left|1-\left(1-{ }_{0} \Phi_{q}(t)\right)_{q}^{(\alpha)}\right| t_{0} d_{q} t\right] \text {, } \\
& \delta_{7}=\left[\int_{\frac{1}{[\alpha+1]_{q}}}^{1}\left|-\left(1-{ }_{0} \Phi_{q}(t)\right)_{q}^{(\alpha)}\right|(1-t)_{0} d_{q} t\right] \text {, } \\
& \delta_{8}=\left[\int_{\frac{1}{[\alpha+1]_{q}}}^{1}\left|-\left(1-{ }_{0} \Phi_{q}(t)\right)_{q}^{(\alpha)}\right| t_{0} d_{q} t\right],
\end{aligned}
$$

which appeared in [40]. 
Theorem 3.5 Let $f:[a, b] \rightarrow \mathbb{R}$ be a continuous function, $\alpha>0$ and ${ }_{a} D_{p, q} f$ be $(p, q)$ integrable on $\left(a, \frac{1}{p}(b-a)+a\right)$. If $\left.\left.\right|_{a} D_{p, q} f\right|^{r}$ is convex on $\left(a, \frac{1}{p}(b-a)+a\right)$ for $r \geq 0$, then the following Riemann-Liouville fractional $(p, q)$-midpoint type inequality holds:

$$
\begin{aligned}
& \left|f\left(\frac{\left.(\alpha+1]_{p, q}-p^{\alpha}\right) a+p^{\alpha} b}{[\alpha+1]_{p, q}}\right)-\frac{\Gamma_{p, q}(\alpha+1)}{p^{\alpha^{2}}(b-a)^{\alpha}}\left(a I_{p, q}^{\alpha} f\right)\left(p^{\alpha} b+\left(1-p^{\alpha}\right) a\right)\right| \\
& \leq(b-a)\left[B_{9}^{1-1 / r}\left(\left.\left.B_{5}\right|_{a} D_{p, q} f(a)\right|^{r}+\left.\left.B_{6}\right|_{a} D_{p, q} f(b)\right|^{r}\right)^{1 / r}\right. \\
& \left.\quad+B_{10}^{1-1 / r}\left(\left.\left.B_{7}\right|_{a} D_{p, q} f(a)\right|^{r}+\left.\left.B_{8}\right|_{a} D_{p, q} f(b)\right|^{r}\right)^{1 / r}\right],
\end{aligned}
$$

where $B_{5}, B_{6}, B_{7}$, and $B_{8}$ are given in Theorem 3.4 and

$$
B_{9}=\int_{0}^{\frac{p^{\alpha}}{(\alpha+1]_{p, q}}}\left|1-\frac{\left(1-{ }_{0} \Phi_{q}(t)\right)_{p, q}^{(\alpha)}}{p^{\left(\begin{array}{c}
\alpha \\
2
\end{array}\right)}}\right| 0 d_{p, q} t
$$

and

$$
B_{10}=\int_{\frac{p^{\alpha}}{[\alpha+1] p, q}}^{1}\left|-\frac{\left(1-{ }_{0} \Phi_{q}(t)\right)_{p, q}^{(\alpha)}}{p^{(\alpha)}\left(\begin{array}{c}
\alpha \\
2
\end{array}\right.}\right|{ }_{0} d_{p, q} t .
$$

Proof Using Lemma 3.2, the power mean inequality and the convexity of $\left|{ }_{a} D_{p, q} f\right|^{r}$, we have

$$
\begin{aligned}
& \left|f\left(\frac{\left([\alpha+1]_{p, q}-p^{\alpha}\right) a+p^{\alpha} b}{[\alpha+1]_{p, q}}\right)-\frac{\Gamma_{p, q}(\alpha+1)}{p^{\alpha^{2}}(b-a)^{\alpha}}\left(a_{p, q}^{\alpha} f\right)\left(p^{\alpha} b+\left(1-p^{\alpha}\right) a\right)\right| \\
& \leq(b-a)\left[\int_{0}^{\frac{p^{\alpha}}{[\alpha+1]_{p, q}}}\left|1-\frac{\left(1-{ }_{0} \Phi_{q}(t)\right)_{p, q}^{(\alpha)}}{p^{\left(\begin{array}{c}
\alpha \\
2
\end{array}\right)}}\right|\left|{ }_{a} D_{p, q} f((1-t) a+t b)\right|{ }_{0} d_{p, q} t\right. \\
& \left.+\int_{\frac{p^{\alpha}}{[\alpha+1 p, q}}^{1}\left|-\frac{\left(1-{ }_{0} \Phi_{q}(t)\right)_{p, q}^{(\alpha)}}{p^{\left(\begin{array}{c}
\alpha \\
2
\end{array}\right)}}\right|{ }_{a} D_{p, q} f((1-t) a+t b) \mid{ }_{0} d_{p, q} t\right] \\
& \leq(b-a)\left[\left(\int_{0}^{\frac{p^{\alpha}}{\alpha+1]_{p, q}}}\left|1-\frac{\left(1-{ }_{0} \Phi_{q}(t)\right)_{p, q}^{(\alpha)}}{p^{\left(\begin{array}{c}
\alpha \\
2
\end{array}\right)}}\right|{ }_{0} d_{p, q} t\right)^{1-1 / r}\right. \\
& \times\left(\left.\int_{0}^{\frac{p^{\alpha}}{(\alpha+1]_{p, q}}}\left|1-\frac{\left(1-{ }_{0} \Phi_{q}(t)\right)_{p, q}^{(\alpha)}}{p^{\left(\frac{\alpha}{2}\right)}}\right|{ }_{a} D_{p, q} f((1-t) a+t b)\right|^{r}{ }_{0} d_{p, q} t\right)^{1 / r} \\
& +\left(\int_{\frac{p^{\alpha}}{[\alpha+1] p, q}}^{1}\left|-\frac{\left(1-{ }_{0} \Phi_{q}(t)\right)_{p, q}^{(\alpha)}}{p^{\left(\begin{array}{c}
\alpha \\
2
\end{array}\right)}}\right|{ }_{0} d_{p, q} t\right)^{1-1 / r} \\
& \left.\times\left(\left.\int_{\frac{p^{\alpha}}{[\alpha+1], q}}^{1}\left|-\frac{\left(1-{ }_{0} \Phi_{q}(t)\right)_{p, q}^{(\alpha)}}{p^{\left(\frac{\alpha}{2}\right)}}\right|{ }_{a} D_{p, q} f((1-t) a+t b)\right|^{r}{ }_{0} d_{p, q} t\right)^{1 / r}\right] \\
& \leq(b-a)\left[\left(\int_{0}^{\frac{p^{\alpha}}{[\alpha+1 \mid p, q}}\left|1-\frac{\left(1-{ }_{0} \Phi_{q}(t)\right)_{p, q}^{(\alpha)}}{p^{\left(\begin{array}{c}
\alpha \\
2
\end{array}\right)}}\right| 0 d_{p, q} t\right)^{1-1 / r}\right. \\
& \times\left(\int_{0}^{\frac{p^{\alpha}}{\alpha+1]_{p, q}}}\left|1-\frac{\left(1-{ }_{0} \Phi_{q}(t)\right)_{p, q}^{(\alpha)}}{p^{\left(\begin{array}{c}
\alpha \\
2
\end{array}\right)}}\right|\left[\left|{ }_{a} D_{p, q} f(a)\right|^{r}(1-t)+\left|{ }_{a} D_{p, q} f(b)\right|^{r} t\right]_{0} d_{p, q} t\right)^{1 / r} \\
& +\left(\int_{\frac{p^{\alpha}}{[\alpha+1] p, q}}^{1}\left|-\frac{\left(1-{ }_{0} \Phi_{q}(t)\right)_{p, q}^{(\alpha)}}{p^{(\alpha)}\left(\begin{array}{c}
\alpha \\
2
\end{array}\right.}\right|{ }_{0} d_{p, q} t\right)^{1-1 / r}
\end{aligned}
$$




$$
\begin{aligned}
& \left.\times\left(\int_{\frac{p^{\alpha}}{[\alpha+1]_{p, q}}}^{1}\left|-\frac{\left(1-{ }_{0} \Phi_{q}(t)\right)_{p, q}^{(\alpha)}}{p^{\left(\begin{array}{c}
\alpha \\
2
\end{array}\right)}}\right|\left[\left|{ }_{a} D_{p, q} f(a)\right|^{r}(1-t)+\left.{ }_{a} D_{p, q} f(b)\right|^{r} t\right]_{0} d_{p, q} t\right)^{1 / r}\right] \\
& \leq(b-a)\left[\left(\int_{0}^{\frac{p^{\alpha}}{[\alpha+1] p, q}}\left|1-\frac{\left(1-{ }_{0} \Phi_{q}(t)\right)_{p, q}^{(\alpha)}}{p^{\left(\begin{array}{c}
\alpha \\
2
\end{array}\right)}}\right|{ }_{0} d_{p, q} t\right)^{1-1 / r}\right. \\
& \times\left(\left|{ }_{a} D_{p, q} f(a)\right|^{r} \int_{0}^{\frac{p^{\alpha}}{\alpha+1 \mid 1 p, q}}\left|1-\frac{\left(1-{ }_{0} \Phi_{q}(t)\right)_{p, q}^{(\alpha)}}{p^{\left(\frac{\alpha}{2}\right)}}\right|(1-t)_{0} d_{p, q} t\right. \\
& \left.\left.+\left|{ }_{a} D_{p, q} f(b)\right|^{r} \int_{0}^{\frac{p^{\alpha}}{\alpha+1]_{p, q}}}\left|1-\frac{\left(1-{ }_{0} \Phi_{q}(t)\right)_{p, q}^{(\alpha)}}{p^{\left(\begin{array}{c}
\alpha \\
2
\end{array}\right)}}\right| t_{0} d_{p, q} t\right)^{1 / r}\right] \\
& +(b-a)\left[\left(\int_{\frac{p^{\alpha}}{[\alpha+1 p, q}}^{1}\left|-\frac{\left(1-{ }_{0} \Phi_{q}(t)\right)_{p, q}^{(\alpha)}}{p^{\left(\begin{array}{c}
\alpha \\
2
\end{array}\right)}}\right|{ }_{0} d_{p, q} t\right)^{1-1 / r}\right. \\
& \times\left(\left|{ }_{a} D_{p, q} f(a)\right|^{r} \int_{\frac{p^{\alpha}}{[\alpha+1] p, q}}^{1}\left|-\frac{\left(1-{ }_{0} \Phi_{q}(t)\right)_{p, q}^{(\alpha)}}{p^{\left(\begin{array}{c}
\alpha \\
2
\end{array}\right)}}\right|(1-t)_{0} d_{p, q} t\right. \\
& \left.\left.+\left|{ }_{a} D_{p, q} f(b)\right|^{r} \int_{\frac{p^{\alpha}}{[\alpha+1] p, q}}^{1}\left|-\frac{\left(1-{ }_{0} \Phi_{q}(t)\right)_{p, q}^{(\alpha)}}{p^{\left(\frac{\alpha}{2}\right)}}\right| t_{0} d_{p, q} t\right)^{1 / r}\right] .
\end{aligned}
$$

This completes the proof.

Remark 3.7 If $\alpha=1$, then (3.24) reduces to

$$
\begin{aligned}
& \left|f\left(\frac{q a+p b}{p+q}\right)-\frac{1}{p(b-a)} \int_{a}^{p b+(1-p) a} f(x)_{a} d_{p, q} x\right| \\
& \leq q(b-a)\left(\frac{p^{2}}{(p+q)^{3}}\right)^{1-1 / r}\left[\left(\left.\left.\lambda_{4}(p, q)\right|_{a} D_{p, q} f(a)\right|^{r}+\left.\lambda_{5}(p, q)\right|_{a} D_{p, q} f(b) \mid r\right)^{1 / r}\right. \\
& \left.\quad+\left(\left.\left.\lambda_{6}(p, q)\right|_{a} D_{p, q} f(a)\right|^{r}+\left.\left.\lambda_{7}(p, q)\right|_{a} D_{p, q} f(b)\right|^{r}\right)^{1 / r}\right],
\end{aligned}
$$

where $\lambda_{4}(p, q), \lambda_{5}(p, q), \lambda_{6}(p, q)$, and $\lambda_{7}(p, q)$ are given in Remark (3.6), which appeared in [42].

Moreover, if $p=1$, then (3.24) reduces to

$$
\begin{gathered}
\left|f\left(\frac{\left([\alpha+1]_{q}-1\right) a+b}{[\alpha+1]_{q}}\right)-\frac{\Gamma_{q}(\alpha+1)}{(b-a)^{\alpha}}\left(a_{a} I_{q}^{\alpha} f\right)(b)\right| \\
\leq(b-a)\left[\delta_{9}^{1-1 / r}\left(\delta_{5}\left|{ }_{a} D_{q} f(a)\right|^{r}+\delta_{6}\left|{ }_{a} D_{q} f(b)\right|^{r}\right)^{1 / r}\right. \\
\left.\quad+\delta_{10}^{1-1 / r}\left(\left.\left.\delta_{7}\right|_{a} D_{q} f(a)\right|^{r}+\delta_{8}\left|{ }_{a} D_{q} f(b)\right|^{r}\right)^{1 / r}\right],
\end{gathered}
$$

where $\delta_{5}, \delta_{6}, \delta_{7}$, and $\delta_{8}$ are given in Remark (3.6) and

$$
\begin{aligned}
& \delta_{9}=\int_{0}^{\frac{1}{\alpha+1]_{q}}}\left|1-\left(1-{ }_{0} \Phi_{q}(t)\right)_{q}^{(\alpha)}\right|{ }_{0} d_{q} t, \\
& \delta_{10}=\int_{\frac{1}{[\alpha+1]_{q}}}^{1}\left|-\left(1-{ }_{0} \Phi_{q}(t)\right)_{q}^{(\alpha)}\right|{ }_{0} d_{q} t,
\end{aligned}
$$

which appeared in [40]. 
Theorem 3.6 Let $f:[a, b] \rightarrow \mathbb{R}$ be a continuous function, $\alpha>0$, and ${ }_{a} D_{p, q} f$ be $(p, q)$ integrable on $\left(a, \frac{1}{p}(b-a)+a\right)$. If $\left|{ }_{a} D_{p, q} f\right|^{r}$ is convex on $\left[a, \frac{1}{p}(b-a)+a\right]$ for $r>1$ and $1 / r+1 / s=1$, then the following Riemann-Liouville fractional $(p, q)$-midpoint type inequality holds:

$$
\begin{aligned}
\mid f( & \left.\frac{\left([\alpha+1]_{p, q}-p^{\alpha}\right) a+p^{\alpha} b}{[\alpha+1]_{p, q}}\right)-\frac{\Gamma_{p, q}(\alpha+1)}{p^{\alpha^{2}}(b-a)^{\alpha}}\left(a^{\alpha} I_{p, q}^{\alpha} f\right)\left(p^{\alpha} b+\left(1-p^{\alpha}\right) a\right) \mid \\
\leq & (b-a)\left[( B _ { 1 1 } ) ^ { 1 / s } \left(\left|{ }_{a} D_{p, q} f(a)\right|^{r}\left(\frac{p^{\alpha}(p+q)[\alpha+1]_{p, q}-p^{\alpha}}{(p+q)\left([\alpha+1]_{p, q}\right)^{2}}\right)\right.\right. \\
& \left.\left.+\left|{ }_{a} D_{p, q} f(b)\right|^{r}\left(\frac{p^{\alpha}}{(p+q)\left([\alpha+1]_{p, q}\right)^{2}}\right)\right)^{1 / r}\right] \\
& +(b-a)\left[( B _ { 1 2 } ) ^ { 1 / s } \left(\left|{ }_{a} D_{p, q} f(a)\right|^{r}\left(\frac{p+q-1}{p+q}-\frac{p^{\alpha}(p+q)[\alpha+1]_{p, q}-p^{2 \alpha}}{(p+q)\left([\alpha+1]_{p, q}\right)^{2}}\right)\right.\right. \\
& \left.\left.+\left|{ }_{a} D_{p, q} f(b)\right|^{r}\left(\frac{1}{p+q}-\frac{p^{2 \alpha}}{(p+q)\left([\alpha+1]_{p, q}\right)^{2}}\right)\right)^{1 / r}\right],
\end{aligned}
$$

where

$$
B_{11}=\int_{0}^{\frac{p^{\alpha}}{[\alpha+1]_{p, q}}}\left|1-\frac{\left(1-{ }_{0} \Phi_{q}(t)\right)_{p, q}^{(\alpha)}}{p^{\left(\begin{array}{c}
\alpha \\
2
\end{array}\right)}}\right|^{s}{ }_{0} d_{p, q} t
$$

and

$$
B_{12}=\int_{\frac{p^{\alpha}}{[\alpha+1]_{p, q}}}^{1}\left|-\frac{\left(1-{ }_{0} \Phi_{q}(t)\right)_{p, q}^{(\alpha)}}{p^{\left(\begin{array}{c}
\alpha \\
2
\end{array}\right)}}\right|^{s}{ }_{0} d_{p, q} t .
$$

Proof Applying Lemma 3.2, Hölder's inequality, and the convexity of $\left.\left.\right|_{a} D_{p, q} f\right|^{r}$, we have

$$
\begin{aligned}
& \left|f\left(\frac{\left([\alpha+1]_{p, q}-p^{\alpha}\right) a+p^{\alpha} b}{[\alpha+1]_{p, q}}\right)-\frac{\Gamma_{p, q}(\alpha+1)}{p^{\alpha^{2}}(b-a)^{\alpha}}\left({ }_{a} I_{p, q}^{\alpha} f\right)\left(p^{\alpha} b+\left(1-p^{\alpha}\right) a\right)\right| \\
& \leq(b-a)\left[\int_{0}^{\frac{p^{\alpha}}{[\alpha+1]_{p, q}}}\left|1-\frac{\left(1-{ }_{0} \Phi_{q}(t)\right)_{p, q}^{(\alpha)}}{p^{\left(\begin{array}{c}
\alpha \\
2
\end{array}\right)}}\right|\left|{ }_{a} D_{p, q} f((1-t) a+t b)\right|_{0} d_{p, q} t\right. \\
& \left.+\int_{\frac{p^{\alpha}}{[\alpha+1]_{p, q}}}^{1}\left|-\frac{\left(1-{ }_{0} \Phi_{q}(t)\right)_{p, q}^{(\alpha)}}{p^{\left(\begin{array}{c}
\alpha \\
2
\end{array}\right)}}\right|\left|{ }_{a} D_{p, q} f((1-t) a+t b)\right|{ }_{0} d_{p, q} t\right] \\
& \leq(b-a)\left[\left(\int_{0}^{\frac{p^{\alpha}}{[\alpha+1]_{p, q}}}\left|1-\frac{\left(1-{ }_{0} \Phi_{q}(t)\right)_{p, q}^{(\alpha)}}{p^{\left(\begin{array}{c}
\alpha \\
2
\end{array}\right)}}\right|^{p}{ }_{0}^{p} d_{p, q} t\right)^{1 / p}\right. \\
& \times\left(\int_{0}^{\frac{p^{\alpha}}{[\alpha+1]_{p, q}}}\left|{ }_{a} D_{p, q} f((1-t) a+t b)\right|^{r}{ }_{0} d_{p, q} t\right)^{1 / r} \\
& +\left(\int_{\frac{p^{\alpha}}{[\alpha+1]_{p, q}}}^{1}\left|-\frac{\left(1-{ }_{0} \Phi_{q}(t)\right)_{p, q}^{(\alpha)}}{p^{\left(\begin{array}{c}
\alpha \\
2
\end{array}\right)}}\right|^{p}{ }_{0} d_{p, q} t\right)^{1 / p} \\
& \left.\times\left(\int_{\frac{p^{\alpha}}{[\alpha+1]_{p, q}}}^{1}\left|{ }_{a} D_{p, q} f((1-t) a+t b)\right|^{r}{ }_{0} d_{p, q} t\right)^{1 / r}\right]
\end{aligned}
$$




$$
\begin{aligned}
& \leq(b-a)\left[\left(\int_{0}^{\frac{p^{\alpha}}{\alpha+1 \mid p, q}}\left|1-\frac{\left(1-{ }_{0} \Phi_{q}(t)\right)_{p, q}^{(\alpha)}}{p^{\left(\begin{array}{c}
\alpha \\
2
\end{array}\right)}}\right|^{p}{ }_{0} d_{p, q} t\right)^{1 / p}\right. \\
& \left.\times\left(\left|{ }_{a} D_{p, q} f(a)\right|^{r} \int_{0}^{\frac{p^{\alpha}}{[\alpha+1]_{p, q}}}(1-t)_{0} d_{p, q} t+\left|{ }_{a} D_{p, q} f(b)\right|^{r} \int_{0}^{\frac{p^{\alpha}}{\alpha+1]_{p, q}}} t_{0} d_{p, q} t\right)^{1 / r}\right] \\
& +(b-a)\left[\left(\int_{\frac{p^{\alpha}}{[\alpha+1] p, q}}^{1}\left|-\frac{\left(1-{ }_{0} \Phi_{q}(t)\right)_{p, q}^{(\alpha)}}{p^{\left(\frac{\alpha}{2}\right)}}\right|^{p}{ }_{0}^{p} d_{p, q} t\right)^{1 / p}\right. \\
& \times\left(\left|{ }_{a} D_{p, q} f(a)\right|^{r} \int_{\frac{p^{\alpha}}{[\alpha+1] p, q}}^{1}(1-t)_{0} d_{p, q} t\right. \\
& \left.\left.+\left|{ }_{a} D_{p, q} f(b)\right|^{r} \int_{\frac{p^{\alpha}}{[\alpha+1] p, q}}^{1} t_{0} d_{p, q} t\right)^{1 / r}\right] \\
& \leq(b-a)\left[\left(\int_{0}^{\frac{p^{\alpha}}{\alpha+1]_{p, q}}}\left|1-\frac{\left(1-{ }_{0} \Phi_{q}(t)\right)_{p, q}^{(\alpha)}}{p^{\left(\frac{\alpha}{2}\right)}}\right|^{p}{ }_{0} d_{p, q} t\right)^{1 / p}\right. \\
& \times\left(\left|{ }_{a} D_{p, q} f(a)\right|^{r}\left(\frac{p^{\alpha}(p+q)[\alpha+1]_{p, q}-p^{\alpha}}{(p+q)\left([\alpha+1]_{p, q}\right)^{2}}\right)\right. \\
& \left.\left.+\left|{ }_{a} D_{p, q} f(b)\right|^{r}\left(\frac{p^{\alpha}}{(p+q)\left([\alpha+1]_{p, q}\right)^{2}}\right)\right)^{1 / r}\right]
\end{aligned}
$$

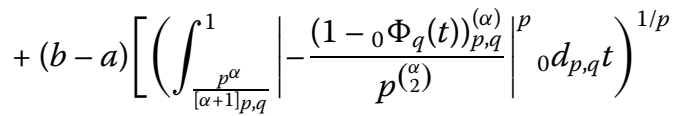

$$
\begin{aligned}
& \times\left(\left|{ }_{a} D_{p, q} f(a)\right|^{r}\left(\frac{p+q-1}{p+q}-\frac{p^{\alpha}(p+q)[\alpha+1]_{p, q}-p^{2 \alpha}}{(p+q)\left([\alpha+1]_{p, q}\right)^{2}}\right)\right. \\
& \left.\left.+\left|{ }_{a} D_{p, q} f(b)\right|^{r}\left(\frac{1}{p+q}-\frac{p^{2 \alpha}}{(p+q)\left([\alpha+1]_{p, q}\right)^{2}}\right)\right)^{1 / r}\right] \text {. }
\end{aligned}
$$

This completes the proof.

Remark 3.8 If $\alpha=1$, then (3.27) reduces to

$$
\begin{aligned}
& \left|f\left(\frac{q a+p b}{p+q}\right)-\frac{1}{p(b-a)} \int_{a}^{p b+(1-p) a} f(x)_{a} d_{p, q} x\right| \\
& \leq q(b-a)\left[( ( \frac { p } { p + q } ) ^ { s + 1 } ( \frac { p - q } { p ^ { s + 1 } - q ^ { s + 1 } } ) ) ^ { 1 / s } \left(\left|{ }_{a} D_{p, q} f(a)\right|^{r}\left(\frac{p^{3}+2 p^{2} q+p q^{2}-p^{2}}{(p+q)^{3}}\right)\right.\right. \\
& \left.\quad+\left|{ }_{a} D_{p, q} f(b)\right|^{r}\left(\frac{p^{2}}{(p+q)^{3}}\right)\right)^{1 / r} \\
& \quad+\left(\int_{\frac{p}{p+q}}^{1}\left(\frac{1}{q}-t\right)^{s}{ }_{0} d_{p, q} t\right)^{1 / s}\left(\left|{ }_{a} D_{p, q} f(b)\right|^{r}\left(\frac{2 p q+q^{2}}{(p+q)^{3}}\right)\right. \\
& \left.\left.\quad+\left.\left.\right|_{a} D_{p, q} f(a)\right|^{r}\left(\frac{p^{2} q+2 p q^{2}-2 p q-q^{2}+q^{3}}{(p+q)^{3}}\right)\right)^{1 / r}\right],
\end{aligned}
$$

which appeared in [42]. 
Moreover, if $p=1$, then (3.27) reduces to

$$
\begin{aligned}
& \left|f\left(\frac{\left([\alpha+1]_{q}-1\right) a+b}{[\alpha+1]_{q}}\right)-\frac{\Gamma_{q}(\alpha+1)}{(b-a)^{\alpha}}\left({ }_{a} I_{q}^{\alpha} f\right)(b)\right| \\
& \leq(b-a)\left[( \delta _ { 1 1 } ) ^ { 1 / s } \left(\left|{ }_{a} D_{q} f(a)\right|^{r}\left(\frac{(1+q)[\alpha+1]_{q}-1}{(1+q)\left([\alpha+1]_{q}\right)^{2}}\right)\right.\right. \\
& \left.\left.\quad+\left.\left.\right|_{a} D_{q} f(b)\right|^{r}\left(\frac{1}{(1+q)\left([\alpha+1]_{q}\right)^{2}}\right)\right)^{1 / r}\right] \\
& \quad+(b-a)\left[( \delta _ { 1 2 } ) ^ { 1 / s } \left(\left|{ }_{a} D_{q} f(a)\right|^{r}\left(\frac{q}{1+q}-\frac{(1+q)[\alpha+1]_{q}-1}{(1+q)\left([\alpha+1]_{q}\right)^{2}}\right)\right.\right. \\
& \left.\left.\quad+\left|{ }_{a} D_{q} f(b)\right|^{r}\left(\frac{1}{1+q}-\frac{1}{(1+q)\left([\alpha+1]_{q}\right)^{2}}\right)\right)^{1 / r}\right]
\end{aligned}
$$

where

$$
\delta_{11}=\int_{0}^{\frac{1}{[\alpha+1]_{q}}}\left|1-\left(1-{ }_{0} \Phi_{q}(t)\right)_{q}^{(\alpha)}\right|^{s}{ }_{0} d_{q} t
$$

and

$$
\delta_{12}=\int_{\frac{1}{[\alpha+1]_{q}}}^{1}\left|-\left(1-{ }_{0} \Phi_{q}(t)\right)_{q}^{(\alpha)}\right|^{s}{ }_{0} d_{q} t,
$$

which appeared in [40].

\section{Conclusions}

In this work, we studied two identities for continuous functions in the form of fractional Riemann-Liouville $(p, q)$-integral. Based on these two identities, some fractional Riemann-Liouville $(p, q)$-trapezoid and $(p, q)$-midpoint type inequalities are given. From this idea, as well as the techniques of this paper, we hope that it will inspire interested readers working in this field.

\section{Acknowledgements}

This work is supported by the Program Management Unit for Human Resources \& Institutional Development, Research and Innovation [grant number B05F630104] and Chiang Mai University, Thailand.

\section{Funding}

Not applicable.

Availability of data and materials

Not applicable.

\section{Competing interests}

The authors declare that they have no competing interests.

\section{Authors' contributions}

All authors contributed equally to this article. They read and approved the final manuscript.

\section{Author details}

${ }^{1}$ Department of Mathematics, Khon Kaen University, 40002 Khon Kaen, Thailand. ${ }^{2}$ Department of Mathematics, Faculty of Applied Science, King Mongkut's University of Technology North Bangkok, 10800 Bangkok, Thailand. ${ }^{3}$ Department of Mathematics, University of loannina, 45110 loannina, Greece. ${ }^{4}$ Nonlinear Analysis and Applied Mathematics (NAAM) - Research Group, Department of Mathematics, Faculty of Science, King Abdulaziz University, 21588 Jeddah, Saudi Arabia. ${ }^{5}$ International Center for Basic and Applied Sciences, 302029 Jaipur, India. ${ }^{6}$ Department of Mathematics, Anand International College of Engineering, 302029 Jaipur, India. 


\section{Publisher's Note}

Springer Nature remains neutral with regard to jurisdictional claims in published maps and institutional affiliations.

\section{Received: 9 April 2021 Accepted: 3 July 2021 Published online: 13 July 2021}

\section{References}

1. Jackson, F.H.: On a q-definite integrals. Q. J. Pure Appl. Math. 41, 193-203 (1910)

2. Jackson, F.H.: q-Difference equations. Am. J. Math. 32, 305-314 (1910)

3. Fock, V: Zur theorie des wasserstoffatoms. Z. Phys. 98, 145-154 (1935)

4. Bangerezaka, G.: Variational q-calculus. J. Math. Anal. Appl. 289, 650-665 (2004)

5. Asawasamrit, S., Sudprasert, C., Ntouyas, S., Tariboon, J.: Some results on quantum Hanh integral inequalities. J. Inequal. Appl. 2019, 154 (2019)

6. Bangerezako, G.: Variational calculus on q-nonuniform. J. Math. Anal. Appl. 306, 161-179 (2005)

7. Exton, H.: q-Hypergeometric Functions and Applications. Halsted, New York (1983)

8. Annyby, H.M., Mansour, S.K.: q-Fractional Calculus and Equations. Springer, Berlin (2012)

9. Ernst, T:. A Comprehensive Treatment of $q$-Calculus. Springer, Basel (2012)

10. Ernst, T: A history of $q$-calculus and a new method. UUDM Report 2000:16, Department of Mathematics, Uppsala University (2000)

11. Ferreira, R.: Nontrivial solutions for fractional $q$-difference boundary value problems. Electron. J. Qual. Theory Differ. Equ. 2010, 70 (2010)

12. Aslam, M., Awan, M.U., Noor, K.I: Quantum Ostrowski inequalities for $q$-differentiable convex function. J. Math. Inequal. 10, 1013-1018 (2016)

13. Aral, A., Gupta, V., Agarwal, R.P.: Applications of q-Calculus in Operator Theory. Springer, New York (2013)

14. Gauchman, H.: Integral inequalities in q-calculus. J. Comput. Appl. Math. 47, 281-300 (2002)

15. Kunt, M., Aljasem, M.: Riemann-Liouville fractional quantum Hermite-Hadamard type inequalities for convex functions. Konuralp J. Math. 8, 122-136 (2020)

16. Dobrogowska, A., Odzijewicz, A.: A second order q-difference equation solvable by factorization method. J. Comput. Appl. Math. 193, 319-346 (2006)

17. Gasper, G., Rahman, M.: Some systems of multivariable orthogonal q-Racah polynomials. Ramanujan J. 13, 389-405 (2007)

18. Ismail, M.E.H., Simeonov, P.: q-Difference operators for orthogonal polynomials. J. Comput. Appl. Math. 233, 749-761 (2009)

19. Bohner, M. Guseinov, G.S.: The $h$-Laplace and $q$-Laplace transforms. J. Comput. Appl. Math. 365, 75-92 (2010)

20. El-Shahed, M., Hassan, H.A.: Positive solutions of $q$-difference equation. Proc. Am. Math. Soc. 138, 1733-1738 (2010)

21. Ahmad, B.: Boundary-value problems for nonlinear third-order q-difference equations. Electron. J. Differ. Equ. 2011, 94 (2011)

22. Ahmad, B., Alsaedi, A., Ntouyas, S.K.: A study of second-order q-difference equations with boundary conditions. Adv. Differ. Equ. 2012, 35 (2012)

23. Ahmad, B., Ntouyas, S.K., Purnaras, I.K.: Existence results for nonlinear q-difference equations with nonlocal boundary conditions. Commun. Appl. Nonlinear Anal. 19, 59-72 (2012)

24. Ahmad, B., Nieto, J.J.: On nonlocal boundary value problems of nonlinear $q$-difference equation. Adv. Differ. Equ. 2012,81 (2012)

25. Kac, V., Cheung, P.: Quantum Calculus. Springer, New York (2002)

26. Noor, M.A., Noor, K.I, Awan, M.U.: Some quantum estimate for Hermite Hadamard inequalities. Appl. Math. Comput. $251,675-679(2015)$

27. Necmettin, A., Mehmet, Z.S., Mehmet, K., Imdat, I.: q-Hermite Hadamard inequalities and quantum estimates for midpoint type inequalities via convex functions and quasi-convex functions. J. King Saud Univ., Sci. 30, 193-203 (2018)

28. Sudsudat, W., Ntouyas, S.K., Tariboon, J.: Quantum integral inequalities for convex functions. J. King Saud Univ., Sci. 9 781-793 (2015)

29. Muhammad, A.N., Khalida, I.N., Muhammad, U.A.: Some quantum integral inequalities via preinvex functions. Appl. Math. Comput. 269, 242-251 (2015)

30. Muhammad, A.N., Muhammad, U.A., Khalida, I.N.: Quantum Ostrowski inequalities for q-differentiable convex functions. Appl. Math. Comput. 10, 1013-1018 (2016)

31. Muhammad, A.K., Noor, M., Eze, N.R., Yu-Ming, C.: Quantum Hermite-Hadamard inequality by means of a Green function. Adv. Differ. Equ. 2020, 99 (2020)

32. Muhammad, A.N., Khalida, I.N., Muhammad, U.A.: Quantum analogues of Hermite-Hadamard type inequalities for generalized convexity. In: Computation, Cryptography, and Network Security, pp. 413-439. Springer, Cham (2015)

33. Humaira, K., Muhammad, I., Baleanu, D., Yu-Ming, C.: New estimates of $q_{1} q_{2}$-Ostrowski-type inequalities within a class of $n$-polynomial prevexity of functions. J. Funct. Spaces 2020, Article ID 3720798 (2020)

34. Wenjun, L., Hefeng, Z:: Some quantum estimates of Hermite-Hadamard inequalities for convex functions. Appl. Math Comput. 251, 675-679 (2015)

35. Tun, M., Gov, E., Balgeçti, S.: Simpson type quantum integral inequalities for convex functions. Miskolc Math. Notes $\mathbf{9}$, 649-664 (2018)

36. Latif, M.A., Dragomir, S.S., Momoniat, E.: Some $\varphi$-analogues of Hermite-Hadamard inequality for s-convex functions in the second sense and related estimates. Punjab Univ. J. Math. 48, 147-166 (2016)

37. Hefeng, Z., Wenjun, L., Jaekeun, P.: Some quantum estimate of Hermite-Hadamard inequalities for quasi-convex functions. Mathematics 7, 152 (2019)

38. Tariboon, J., Ntouyas, S.K., Agarwal, P.: New concepts of fractional quantum calculus and applications to impulsive fractional q-difference equations. Adv. Differ. Equ. 2015, 18 (2015)

39. Sudsutad, W., Ntouyas, S.K., Tariboon, J.: Integral inequalities via fractional quantum calculus. J. Inequal. Appl. 2016, 81 (2016) 
40. Kunt, M., Aljasem, M.: Fractional quantum Hermite-Hadamard type inequalities. Konuralp J. Math. 8, 122-136 (2020)

41. Neang, P., Nonlaopon, K., Tariboon, J., Ntouyas, S.K.: Fractional $(p, q)$-calculus on finite intervals and some integral inequalities. Symmetry 13, 504 (2021)

42. Kunt, M., İşcan, I., Alp, N., Sarakaya, M.Z.: (p, q)-Hermite-Hadamard inequalities and $(p, q)$-estimates for midpoint type inequalities via convex and quasi-convex functions. Rev. R. Acad. Cienc. 112, 969-992 (2018)

43. Latif, M.A., Kunt, M., Dragomir, S.S., Isscan, I.: (p,q)-Trapezoid type inequalities. Rev. R. Acad. Cienc. 112, 969-992 (2018)

44. Kamsrisuk, N., Promsakon, C., Ntouyas, S.K., Tariboon, J.: Nonlocal boundary value problems for $(p, q)$-difference equations. Differ. Equ. Appl. 10, 183-195 (2018)

45. Nuntigrangjana, T., Putjuso, S., Ntouyas, S.K., Tariboon, J.: Impulsive quantum $(p, q)$-difference equations. Adv. Differ. Equ. 2020, 98 (2020)

46. Promsakon, C., Kamsrisuk, N., Ntouyas, S.K., Tariboon, J.: On the second-order quantum ( $p, q)$-difference equations with separated boundary conditions. Adv. Math. Phys. 2018, Artical ID 9089865 (2018)

47. Chakrabarti, R., Jagannathan, R.: A $(p, q)$-oscillator realization of two-parameter quantum algebras. J. Phys. A, Math. Gen. 24, L711-L718 (1991)

48. Burban, I.: Two-parameter deformation of the oscillator algebra and $(p, q)$-analog of two-dimensional conformal field theory. J. Nonlinear Math. Phys. 2, 384-391 (1995)

49. Burban, I.M., Klimyk, A.U.: $(p, q)$-Differentiation, $(p, q)$-integration, and $(p, q)$-hypergeometric functions related to quantum groups. Integral Transforms Spec. Funct. 2, 15-36 (1994)

50. Hounkonnou, M.N., Désiré, J., Kyemba, B.R.: $(p, q)$-Calculus: differentiation and integration. SUT J. Math. 49, 145-167 (2013)

51. Aral, A., Gupta, V.: Applications of (p, q)-gamma function to Szász Durrmeyer operators. Publ. Inst. Math. 102, 211-220 (2017)

52. Sahai, V., Yadav, S.: Representations of two parameter quantum algebras and ( $p, q)$-special functions. J. Math. Anal. Appl. 335, 268-279 (2007)

53. Sadjang, P.N.: On the fundamental theorem of $(p, q)$-calculus and some $(p, q)$-Taylor formulas. Results Math. 73, 39 (2018)

54. Usman, T., Saif, M., Choi, J.: Certain identities associated with $(p, q)$-binomial coefficients and $(p, q)$-Stirling polynomials of the second kind. Symmetry 12, 1436 (2020)

55. Sadjang, P.N.: On the $(p, q)$-gamma and the $(p, q)$-beta functions. arXiv preprint (2015). arXiv:1506.07394

56. Sadjang, P.N.: On two $(p, q)$-analogues of the Laplace transform. J. Differ. Equ. Appl. 23, 1562-1583 (2017)

57. Mursaleen, M., Ansari, K.J., Khan, A.: On (p, q)-analogues of Bernstein operators. Appl. Math. Comput. 266, 874-882 (2015)

58. Mursaleen, M., Ansari, K.J., Khan, A.: Erratum to "On $(p, q)$-analogues of Bernstein operators". Appl. Math. Comput. 278 70-71 (2016)

59. Kang, S.M., Rafiq, A., Acu, A.M., Ali, F., Kwun, Y.C.: Erratum to "Some approximation properties of (p, q)-Bernstein operators". J. Inequal. Appl. 2016, 169 (2016)

60. Mursaleen, M., Khan, F., Khan, A.: Approximation by $(p, q)$-Lorentz polynomials on a compact disk. Complex Anal. Oper. Theory 10, 1725-1740 (2016)

61. Cai, Q.-B., Zhou, G.: On (p, q)-analogues of Kantorovich type Bernstein-Stancu-Schurer operator. Appl. Math. Comput. 276, 12-20 (2016)

62. Mursaleen, M., Ansari, K.J., Khan, A.: Some approximation results of $(p, q)$-analogues of Bernstein-Stancu operators Appl. Math. Comput. 264, 392-402 (2015)

63. Acar, T., Aral, A., Mohiuddine, S.A.: On Kantorovich modification of (p,q)-Baskakov operators. J. Inequal. Appl. 2016, 98 (2016)

64. Mursaleen, M., Nasiruzzaman, M., Khan, F., Khan, A.: $(p, q)$-Analogues of divided difference and Bernstein operators. J. Nonlinear Funct. Anal. 2017, 25 (2017)

65. Wachs, M., White, D.: (p, q)-Stirling numbers and set partition statistics. J. Comb. Theory, Ser. A 56, 27-46 (1991)

66. Wachs, M.L.: $\sigma$-Restricted growth functions and (p,q)-Stirling numbers. J. Comb. Theory, Ser. A 68, 470-480 (1994)

67. Remmel, J.B., Wachs, M.: Rook theory, generalized Stirling numbers and $(p, q)$-analogues. Electron. J. Comb. 11, R84 (2004)

68. Médics, A.D., Leroux, P.: Generalized Stirling numbers, convolution formula and $(p, q)$-analogues. Can. J. Math. 11, 474-499 (1995)

69. Bukweli-Kyemba, J.D., Hounkonnou, M.N.: Quantum deformed algebra: coherent states and special functions (2013). arXiv:1301.0116v1

70. Prabseang, J., Nonlaopon, K., Tariboon, J.: (p, q)-Hermite-Hadamard inequalities for double integral and $(p, q)$-differentiable convex functions. Axioms 8, 68 (2019)

71. Kalsoom, H., Amer, M., Junjua, M.D., Hassain, S., Shahxadi, G.: (p, q)-Estimates of Hermite-Hadamard-type inequalities for coordinated convex and quasi convex function. Mathematics 7, 683 (2019)

72. Tunc, M., Gov, E.: Some integral inequalities via $(p, q)$-calculus on finite intervals. RGMIA Res. Rep. Collect. 19, 1-12 (2016)

73. Tunc, M., Gov, E.: (p, q)-Integral inequalities. RGMIA Res. Rep. Collect. 19, 1-13 (2016)

74. Soontharanon, J., Sitthiwirattham, T.: Fractional $(p, q)$-calculus. Adv. Differ. Equ. 2020, 35 (2020)

75. Gradimir, V.M., Vijay, G.N.M.: $(p, q)$-Beta functions and applications in approximation. Bol. Soc. Mat. Mex. 24, 219-237 (2018)

76. Dragomir, S.S., Agarwal, R.P.: Two inequalities for differentiable mapping and applications to special means of real numbers and to trapezoidal formula. Appl. Math. Lett. 11,91-95 (1995) 